STRUCTURAL STABILITY OF GENERALIZED FORCHHEIMER EQUATIONS FOR COMPRESSIBLE FLUIDS IN POROUS MEDIA

By

\title{
Luan Hoang
}

and

Akif Ibragimov

IMA Preprint Series \# 2294

( January 2010)

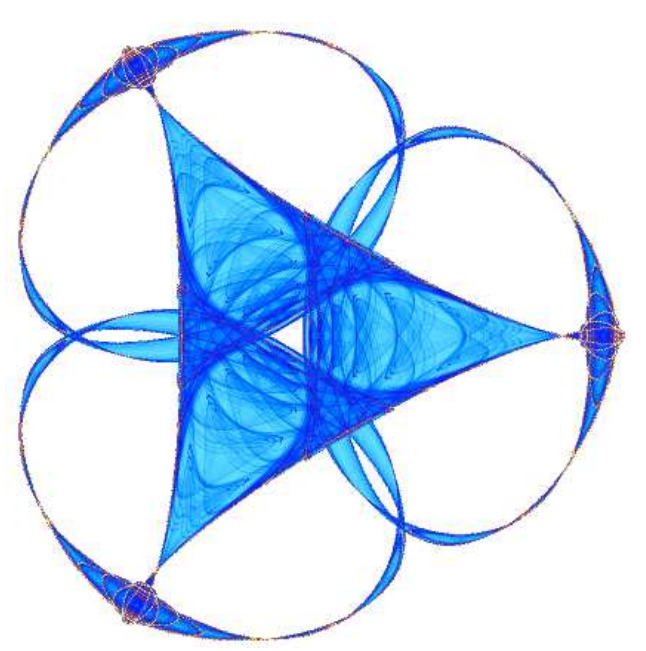

INSTITUTE FOR MATHEMATICS AND ITS APPLICATIONS

UNIVERSITY OF MINNESOTA 400 Lind Hall

207 Church Street S.E.

Minneapolis, Minnesota 55455-0436

Phone: 612-624-6066 Fax: 612-626-7370

URL: http://www.ima.umn.edu 


\title{
STRUCTURAL STABILITY OF GENERALIZED FORCHHEIMER EQUATIONS FOR COMPRESSIBLE FLUIDS IN POROUS MEDIA
}

\author{
LUAN HOANG AND AKIF IBRAGIMOV ${ }^{\dagger}$
}

\begin{abstract}
We study the generalized Forchheimer equations for slightly compressible fluids in porous media. The structural stability is established with respect to either the boundary data or the coefficients of the Forchheimer polynomials. An inhomogeneous Poincare-Sobolev inequality related to the non-linearity of the equation is used to study the asymptotic behavior of the solutions. Moreover, we prove a perturbed monotonicity property of the vector field associated with the resulting non-Darcy equation, where the correction is linear in the coefficients of the Forchheimer polynomials.
\end{abstract}

\section{Contents}

1. Introduction

2. Preliminaries and Auxiliary Results 3

3. Bounds for the Solutions 8

4. Dependence on the Boundary Data 21

4.1. Spatial homogeneous boundary data and their perturbations 21

4.2. Continuous dependence on the boundary data 25

5. Dependence on the Forchheimer Polynomials 31

Appendix A. 38

$\begin{array}{ll}\text { References } & 39\end{array}$

\section{INTRODUCTION}

The Forchheimer equations were introduced to describe the fluid flows in porous media in cases when the Darcy Law does not apply [15, 10, 21]. The classical Forchheimer equations are generalized and used to study an increasing number of nonlinear phenomena in porous media [5, 11, 23]. In [13] non-classical non-linear Forchheimer equations were obtained via homogenization procedure from NavierStokes system defined in the media with periodic geometry. Another approach to non-linear Darcy equations is based on the application of mixture theory [23] to account the inertia forces due to interactions between the fluid and the matrix of porous media. Other arguments for derivation of the non-linear Forchheimer equation are discussed in [22, 25], and in the concluding remarks of [13]. Forchheimer flows in porous media for incompressible fluids is intensively studied within

Date: January 12, 2010.

Key words and phrases. non-linear Darcy, generalized Forchheimer equations, porous media, long-time dynamics, stability. 
context of the Brinkman-Forchheimer model, c.f. [17, 18, 19, 20, 24, 6]. Other numerical studies of classical and generalized Forchheimer equations can be found in $[7,8,9,16]$.

In this work we investigate generalized Forchheimer flows for porous media subjected to mixed boundary conditions. These flows are characterized by the polynomial equation $g(|u|) u=-\nabla p$ relating velocity field $u$ to gradient of the pressure $\nabla p$. This is widely used to match experimental field data and to calculate hydrodynamical parameters of the processes in the porous media by physicists and engineers $[5,4,23,11,3]$. For slightly compressible fluid, the system of equations describing the fluid motion reduces to a scalar equation of pressure function. This is a non-linear parabolic equation, which degenerates as the pressure gradient goes to infinity [1]. This reduction of the original system to parabolic equation enables the investigation of qualitative properties of the corresponding solutions for wide class of the boundary conditions. The essence of our study is understanding the relations between the solution, the non-linearity of the Forchheimer polynomial and the non-homogeneous boundary data by exploring the structure of the the equation.

The first topic of the paper is the the structural stability of the initial boundary value problem (IBVP) with respect to the boundary data. This topic was studied in our previous work [1] for the boundary regime of special type (called (S)) when the permeability inside the domain is much smaller than that on the boundary. Under this constraint, the boundary data are split in time and spatial variables (see [1] for details) for all times. Though the splitting condition presents clear dynamical features of the equation, it is restrictive from theoretical and applied points of view. In this paper the general Dirichlet boundary data on a part of the boundary is considered. The dependence of the solutions on the boundary data is analyzed, particularly, the relations between their asymptotic behaviors.

The second topic is the stability of the hydrodynamical quantities of fluid flows with respect to parameters of the constitutive equations. We establish the quantified continuous dependence of the energy norms of the solutions on the coefficients of the Forchheimer polynomials. Under certain controls of the degree of the Forchheimer polynomials and the related growth conditions on the boundary data the asymptotic deviation between solutions are determined by that between the coefficients.

For these purposes, various a priori estimates for individual solutions need be obtained including those for the Lebesgue norms as well as Sobolev norms in both time and space variables. As in [1], we make use of a Lyapunov-like functional, which is comparable to the Sobolev norms of the solutions. Refined estimates of this functional are obtained. Inhomogeneous Poincare-Sobolev inequalities of a particular weighted form related to the non-linearity of the Forchheimer polynomials are also used (see Lemma 2.4).

The structure of the equation has an important degenerate monotonicity property, which allows comparisons between two solutions via their gradients. A priori bounds for individual solutions in terms of the boundary data are used to control the degeneracy of the monotonicity. This leads to the establishment of the stability with respect to the boundary data. The asymptotic behavior of the solution is uniquely determined by the asymptotic behavior of the boundary data. Moreover, 
for Forchheimer polynomials with varying coefficients, the structure of monotonicity is preserved upto a correction which is comparable to the difference between coefficients of two Forchheimer polynomials (see Lemma 5.2).

The paper is organized as follows. In Sect. 2, we recall main definitions and properties of degenerate parabolic operator. We re-estimate the non-linear permeability functions with the constants having explicit dependence on the coefficients of the Forchheimer polynomials. In Sect. 3, we obtain various a priori estimates for the individual solutions of the IBVP. These include the estimates for spatial and temporal derivatives of the solutions. In case the Forchheimer polynomial has small degree, inhomogeneous Poincare-Sobolev inequality related to the non-linearity of the Forchheimer polynomial is used to obtain estimates of the energy type norms for the pressure function. These estimates are basic for our study of the asymptotic properties of the solutions as $t \rightarrow \infty$. In Sect. 4, we establish the structural stability of the solutions with respect to the boundary data. In Subsection 4.1 we prove the convergence in Sobolev norms with respect to time and space of the solutions with spatial homogeneous boundary data and their perturbations. In Subsect. 4.2, we study the convergence and stability in $L^{2}$-norm of the solutions with general boundary data. Under certain conditions on the long time behavior of the data, the asymptotic and Lyapunov type stability is obtained. It is noteworthy to point out that the boundary data can be unbounded when time is large. In Sect. 5, we show the continuous dependence of the solutions on the Forchheimer polynomials. For this, we prove a perturbed monotonicity property which depends continuously on the coefficients of the Forchheimer polynomials. In the Appendix, we prove an estimate of solutions to a particular differential inequality. This is used to improve main a priori estimates in Sect. 3 .

\section{Preliminaries and Auxiliary Results}

Consider fluid flows with velocity $u(x, t)$, pressure $p(x, t)$ and density $\rho(x, t)$. For porous media, the following empirical relations are studied:

Darcy's law: $\alpha u=-\nabla p$;

Forchheimer's laws: $\alpha u+\beta|u| u=-\nabla p, \alpha u+\beta|u| u+\gamma|u|^{2} u=-\nabla p$, and $\alpha u+\beta|u|^{m-1} u=-\nabla p$.

A generalized Forchheimer equation that covers most applications is studied in [1] and is of the form:

$$
g(|u|) u=-\nabla p,
$$

where $g(s) \geq 0$ is a function defined on $[0, \infty)$.

From (2.1) one can solve $u$ implicitly in terms of $\nabla p$ and derives a non-linear Darcy equation:

$$
u=\frac{-\nabla p}{g\left(G^{-1}(|\nabla p|)\right)}=-K(|\nabla p|) \nabla p,
$$

where $G(s)=s g(s)$ and the function $K: \mathbb{R}_{+} \rightarrow \mathbb{R}_{+}$, where $\mathbb{R}_{+}=[0, \infty)$, is defined by

$$
K(\xi)=\frac{1}{g\left(G^{-1}(\xi)\right)}, \quad \xi \geq 0
$$


Other equations governing the motion of the fluid are the equation of continuity:

$$
\frac{d}{d t} \rho=\nabla \cdot(\rho u)
$$

and the equation of state which, for slightly compressible fluids (cf. [5, 15]), has the following form:

$$
\rho(p)=\rho_{0} \exp \left(\frac{p-p_{0}}{\kappa}\right) \quad \text { or } \quad \frac{d \rho}{d p}=\frac{\rho}{\kappa}, \quad \kappa>0 .
$$

From (2.2), (2.4) and (2.5) one derives a scalar equation for the pressure:

$$
\frac{\partial p}{\partial t}=-\kappa \nabla \cdot(K(|\nabla p|) \nabla p)-K(|\nabla p|)|\nabla p|^{2} .
$$

Since for most slightly compressible fluids in porous media the constant $\kappa$ is large, we neglect the last term in (2.6) and study the reduced degenerate parabolic equation of the form:

$$
\frac{\partial p}{\partial t}=-\kappa \nabla \cdot(K(|\nabla p|) \nabla p)
$$

Note that this reduction is commonly used in engineering.

By changing the reference system, we obtain a non-dimensional equation which is (2.7) with $\kappa=1$.

Among many possible generalizations of the classical Forchheimer equations, we consider the following models which cover most of the applications. In this paper, we study the case when the function $g$ in $(2.1)$ is a generalized polynomial with non-negative coefficients. Specifically, the function $g: \mathbb{R}_{+} \times \mathbb{R}_{+}^{N+1} \rightarrow \mathbb{R}$ is of the form

$$
g(s, \vec{a})=g(s, \vec{a} ; \vec{\alpha})=a_{0} s^{\alpha_{0}}+a_{1} s^{\alpha_{1}}+\ldots+a_{N} s^{\alpha_{N}},
$$

where $\alpha_{0}=0<\alpha_{1}<\ldots<\alpha_{N}$, the coefficients $a_{0}, a_{1}, \ldots, a_{N}$ are non-negative with $a_{0}>0$ and $a_{N}>0$.

The number $\alpha_{N}$ is the degree of $g$ and is denoted by $\operatorname{deg}(g)$. We call $\vec{\alpha}=$ $\left(\alpha_{0}, \ldots, \alpha_{N}\right)$ the exponent vector and $\vec{a}=\left(a_{0}, \ldots, a_{N}\right)$ the coefficient vector. The number $N+1$ is the length of $g$. This class of functions $g(s, \vec{a})$ is denoted by $\operatorname{FP}(N, \vec{\alpha})$, which is the abbreviation of "Forchheimer polynomials".

When the function $g$ in $(2.1)$ belongs to $\operatorname{FP}(N, \vec{\alpha})$, it is referred to as the Forchheimer polynomial of the equation.

Let $N$ and $\vec{\alpha}$ be fixed. Let

$$
R(N)=\left\{\vec{a}=\left(a_{0}, a_{1}, \ldots, a_{N}\right) \subset \mathbb{R}^{N+1}: a_{0}, a_{N}>0, a_{1}, \ldots, a_{N-1} \geq 0\right\} .
$$

Let $g=g(s, \vec{a})$ be in $\operatorname{FP}(N, \vec{\alpha})$ with $\vec{a} \in R(N)$. We denote

$$
\begin{gathered}
a=\frac{\alpha_{N}}{1+\alpha_{N}}, \\
\chi(\vec{a})=\max \left\{1, a_{0}, a_{1}, \ldots, a_{N}, \frac{1}{a_{0}}, \frac{1}{a_{N}}\right\} .
\end{gathered}
$$

Many estimates below will have constants depending on $\chi(\vec{a})$.

For each $\xi \geq 0$, we denote by $s=s(\xi, \vec{a})$ the unique solution of the equation $s g(s, \vec{a})=\xi$, i.e., one has

$$
s(\xi, \vec{a}) g(s(\xi, \vec{a}), \vec{a})=\xi .
$$


The same as (2.3), we define

$$
K(\xi, \vec{a})=\frac{1}{g(s(\xi, \vec{a}), \vec{a})} .
$$

In [1], we have the following properties for $K(\xi, a)$ : it is decreasing in the variable $\xi$ and satisfies

$$
\begin{gathered}
K(\xi, \vec{a}) \leq K(0, \vec{a})=a_{0}^{-1} \leq \chi(\vec{a}), \\
C_{1}(1+\xi)^{-a} \leq K(\xi, \vec{a}) \leq C_{2}(1+\xi)^{-a} .
\end{gathered}
$$

The constants $C_{1}$ and $C_{2}$ above were not computed explicitly in [1]. For our present study, the dependence of those constants on the coefficient vector is important, hence we carefully re-estimate $K(\xi, \vec{a})$ and specify this dependence.

Lemma 2.1. Let $g(s, \vec{a})$ be in class $F P(N, \vec{\alpha})$. One has for any $\xi \geq 0$ that

$$
\frac{C_{0}^{-1} \chi(\vec{a})^{-1-a}}{(1+\xi)^{a}} \leq K(\xi, \vec{a}) \leq \frac{C_{0} \chi(\vec{a})^{1+a}}{(1+\xi)^{a}}
$$

and for any $m \geq 1, \delta>0$ that

$$
C_{0}^{-1} \chi(\vec{a})^{-1-a} \frac{\delta^{a}}{(1+\delta)^{a}}\left(\xi^{m-a}-\delta^{m-a}\right) \leq K(\xi, \vec{a}) \xi^{m} \leq C_{0} \chi(\vec{a})^{1+a} \xi^{m-a} .
$$

where $C_{0}=C_{0}\left(N, \alpha_{N}\right)$ depends on $N, \alpha_{N}$ only.

In particular, when $m=2, \delta=1$, one has

$$
2^{-a} C_{0}^{-1} \chi(\vec{a})^{-1-a}\left(\xi^{2-a}-1\right) \leq K(\xi, \vec{a}) \xi^{2} \leq C_{0} \chi(\vec{a})^{1+a} \xi^{2-a} .
$$

Proof. Let $g(s)=g(s, \vec{a})$ and $K(\xi)=K(\xi, \vec{a})$. On one hand,

$$
\begin{aligned}
g(s)=\sum_{j=0}^{N} a_{j} s^{\alpha_{j}} & \leq \max _{j=0, \ldots, N}\left\{a_{j}\right\}\left(1+\sum_{j=2}^{N}(1+s)^{\alpha_{j}}+s^{\alpha_{N}}\right) \\
& \leq \max _{j=0, \ldots, N}\left\{a_{j}\right\}(N+1)(1+s)^{\alpha_{N}}, \\
g(s)=\sum_{j=0}^{N} a_{j} s^{\alpha_{j}} & \geq a_{0}+a_{N} s^{\alpha_{N}} \geq \min \left\{a_{0}, a_{N}\right\}\left(1+s^{\alpha_{N}}\right) \\
& \geq 2^{-\alpha_{N}} \min \left\{a_{0}, a_{N}\right\}(1+s)^{\alpha_{N}} .
\end{aligned}
$$

On the other hand,

$$
\begin{gathered}
1+\xi=1+s g(s) \leq \max _{j=0, \ldots, N}\left\{1, a_{j}\right\}\left(1+\sum_{j=0}^{N} s^{\alpha_{j}+1}\right) \\
\leq \max _{j=0, \ldots, N}\left\{1, a_{j}\right\}(N+2)(1+s)^{\alpha_{N}+1}, \\
1+\xi=1+s g(s) \geq 1+a_{N} s^{\alpha_{N}+1} \geq 2^{-\alpha_{N}-1} \min \left\{1, a_{N}\right\}(1+s)^{\alpha_{N}+1} .
\end{gathered}
$$

Hence

$$
\begin{aligned}
K(\xi)=\frac{1}{g(s)} & \leq \frac{2^{\alpha_{N}}}{\min \left\{a_{0}, a_{N}\right\}(1+s)^{\alpha_{N}}} \\
& \leq \frac{2^{\alpha_{N}}\left(\max _{j=0, \ldots, N}\left\{1, a_{j}\right\}(N+2)\right)^{\frac{\alpha_{N}}{\alpha_{N}+1}}}{\min \left\{a_{0}, a_{N}\right\}(1+\xi)^{\frac{\alpha_{N}}{\alpha_{N}+1}}}=\frac{C_{2}^{\prime}}{(1+\xi)^{a}}
\end{aligned}
$$




$$
\begin{aligned}
K(\xi)=\frac{1}{g(s)} & \geq \frac{1}{\max _{j=0, \ldots, N}\left\{a_{j}\right\}(N+1)(1+s)^{\alpha_{N}}} \\
& \geq \frac{\left(2^{-\alpha_{N}-1} \min \left\{1, a_{N}\right\}\right)^{\frac{\alpha_{N}}{\alpha_{N}+1}}}{\max _{j=0, \ldots, N}\left\{a_{j}\right\}(N+1)(1+\xi)^{\frac{\alpha_{N}}{\alpha_{N}+1}}}=\frac{C_{1}^{\prime}}{(1+\xi)^{a}},
\end{aligned}
$$

where

$$
C_{1}^{\prime}=\frac{\left(\min \left\{1, a_{N}\right\}\right)^{a}}{2^{\alpha_{N}+1}(N+1) \max _{j=0, \ldots, N}\left\{a_{j}\right\}}, C_{2}^{\prime}=\frac{2^{\alpha_{N}}(N+2)^{a}\left(\max _{j=0, \ldots, N}\left\{1, a_{j}\right\}\right)^{2}}{\min \left\{a_{0}, a_{N}\right\}} .
$$

One easily sees that there is $C_{0}=C_{0}\left(N, \alpha_{N}\right)$ such that

$$
\begin{aligned}
& C_{1}=C_{0}^{-1} \chi(\vec{a})^{-1-a} \leq C_{1}^{\prime} \leq C_{0} \chi(\vec{a}), \\
& C_{0}^{-1} \chi(\vec{a})^{-1} \leq C_{2}^{\prime} \leq C_{0} \chi(\vec{a})^{1+a}=C_{2} .
\end{aligned}
$$

Hence (2.16) follows.

For (2.17), one notes that its second inequality immediately follows (2.16). For its first inequality, one considers two cases:

If $\xi>\delta$ then

$$
\begin{aligned}
K(\xi) \xi^{m} & \geq C_{0}^{-1} \chi(\vec{a})^{-1-a} \xi^{m}(1+\xi)^{-a} \geq C_{0}^{-1} \chi(\vec{a})^{-1-a} \xi^{m}\left(\frac{\xi}{\delta}+\xi\right)^{-a} \\
& =C_{0}^{-1} \chi(\vec{a})^{-1-a}\left(\frac{\delta}{1+\delta}\right)^{a} \xi^{m-a} \geq C_{0}^{-1} \chi(\vec{a})^{-1-a}\left(\frac{\delta}{1+\delta}\right)^{a}\left(\xi^{m-a}-\delta^{m-a}\right) .
\end{aligned}
$$

If $\xi \leq \delta$ then

$$
K(\xi) \xi^{m} \geq 0 \geq C_{0}^{-1} \chi(\vec{a})^{-1-a}\left(\frac{\delta}{1+\delta}\right)^{a}\left(\xi^{m-a}-\delta^{m-a}\right) .
$$

The proof is complete.

It is also proved in Lemmas III.5 and III.9 of [1] that for $\xi \geq 0$, one has

$$
-a K(\xi, \vec{a}) \leq\left(\partial_{\xi} K(\xi, \vec{a})\right) \xi \leq 0 .
$$

Next, we introduce the function $H$ which, roughly speaking, will play the role of a Lyapunov function in our estimates.

Definition 2.2. For any $\xi \geq 0$, one defines

$$
H(\xi, \vec{a})=\int_{0}^{\xi^{2}} K(\sqrt{s}, \vec{a}) d s
$$

The function $H(\xi, \vec{a})$ can be compared with $\xi$ and $K(\xi, \vec{a})$ as follows (see [1]):

$$
\begin{gathered}
K(\xi, \vec{a}) \xi^{2} \leq H(\xi, \vec{a}) \leq 2 K(\xi, \vec{a}) \xi^{2}, \\
H(\xi, \vec{a}) \leq K(0, \vec{a}) \xi^{2}=a_{0}^{-1} \xi^{2} \leq \chi(\vec{a}) \xi^{2} .
\end{gathered}
$$

As a consequence of (2.23) and (2.18), one has:

$$
C_{3}\left(\xi^{2-a}-1\right) \leq H(\xi, \vec{a}) \leq C_{4} \xi^{2-a} .
$$

where $C_{3}$ and $C_{4}$ depend on $N, \alpha_{N}$ and $\chi(\vec{a})$.

Taking into account the explicit estimate $(2.16)$, the monotonicity properties in Proposition III.6 and Lemma III.11 of [1] can now be rewritten as: 
Lemma 2.3 ([1]). (i) For any $y, y^{\prime} \in \mathbb{R}^{n}$, one has

$$
\left(K(|y|, \vec{a}) y-K\left(\left|y^{\prime}\right|, \vec{a}\right) y^{\prime}\right) \cdot\left(y-y^{\prime}\right) \geq a K\left(\max \left\{|y|,\left|y^{\prime}\right|\right\}, \vec{a}\right)\left|y-y^{\prime}\right|^{2} .
$$

(ii) For any functions $p_{1}$ and $p_{2}$ one has

$$
\begin{gathered}
\int_{U}\left(K\left(\left|\nabla p_{1}\right|, \vec{a}\right) \nabla p_{1}-K\left(\left|\nabla p_{2}\right|, \vec{a}\right) \nabla p_{2}\right) \cdot\left(\nabla p_{1}-\nabla p_{2}\right) d x \\
\geq a\left(\int_{U} K\left(\max \left\{\left|\nabla p_{1}\right|,\left|\nabla p_{2}\right|\right\}, \vec{a}\right)\left|\nabla p_{1}-\nabla p_{2}\right|^{2} d x\right) \\
\geq C_{5}\left(\int_{U}\left|\nabla p_{1}-\nabla p_{2}\right|^{2-a} d x\right)^{\frac{2}{2-a}}\left(1+\max \left\{\left\|\nabla p_{1}\right\|_{L^{2-a}(U)},\left\|\nabla p_{2}\right\|_{L^{2-a}(U)}\right\}\right)^{-a},
\end{gathered}
$$

where $C_{5}=C_{5}(N, \operatorname{deg}(g), \chi(\vec{a}))$.

Degree Condition (DC): The following condition on the degree of the Forchheimer polynomial will be vital to our study of long time behavior of the solutions:

$$
\operatorname{deg}(g) \leq \frac{4}{n-2} .
$$

We will refer to it as the Degree Condition.

The following inhomogeneous Sobolev-Poincare inequality of weighted form will be used to obtain a priori estimates of the solution.

Lemma 2.4. Let $f(x)$ and $\xi(x)$ be two functions on $U$ with $f(x)$ vanishing on $\Gamma_{1}$ and $\xi(x) \geq 0$. Then

$\left(\int_{U}|f(x)|^{(2-a)^{*}} d x\right)^{\frac{2}{(2-a)^{*}}} \leq C_{6}\left(\int_{U} K(\xi(x), \vec{a})|\nabla f(x)|^{2} d x\right)\left(1+\int_{U} H(\xi(x), \vec{a}) d x\right)^{\frac{a}{2-a}}$, where $(2-a)^{*}=n(2-a) /(n-(2-a))$ and $C_{6}=C_{6}(N, \operatorname{deg}(g), \chi(\vec{a}), U)$.

Subsequently, when $\operatorname{deg}(g) \leq 4 /(n-2)$ one has

$$
\int_{U}|f(x)|^{2} d x \leq C_{7}\left(\int_{U} K(\xi(x), \vec{a})|\nabla f(x)|^{2} d x\right)\left(1+\int_{U} H(\xi(x), \vec{a}) d x\right)^{\frac{a}{2-a}}
$$

where $C_{7}=C_{7}(N, \operatorname{deg}(g), \chi(\vec{a}), U)$.

Proof. Let $K(\xi)=K(\xi, \vec{a})$ and $H(\xi)=H(\xi, \vec{a})$. Let $r \geq 1$ and $r^{*}=n r /(n-r)$.

Using Poincare-Sobolev inequality ([12]) and then Holder inequality, one derives

$$
\begin{aligned}
\left(\int_{U}|f|^{r^{*}} d x\right)^{\frac{2}{r^{*}}} & \leq C\left(\int_{U}|\nabla f|^{r} d x\right)^{\frac{2}{r}} \\
& =C\left(\int_{U}|\nabla f|^{r} K(\xi)^{\frac{r}{2}} K(\xi)^{-\frac{r}{2}} d x\right)^{\frac{2}{r}} \\
& \leq C\left(\int_{U}|\nabla f|^{2} K(\xi) d x\right)\left(\int_{U} K(\xi)^{-\frac{r}{2} \cdot \frac{2}{2-r}} d x\right)^{\frac{2}{r} \cdot \frac{2-r}{2}} \\
& =C\left(\int_{U}|\nabla f|^{2} K(\xi) d x\right)\left(\int_{U} K(\xi)^{-\frac{r}{2-r}} d x\right)^{\frac{2-r}{r}} .
\end{aligned}
$$

By relations (2.16) and (2.25), it follows that

$$
\left(\int_{U}|f|^{r^{*}} d x\right)^{\frac{2}{r^{*}}} \leq C\left(\int_{U}|\nabla f|^{2} K(\xi) d x\right)\left(\int_{U}(1+\xi)^{a \cdot \frac{r}{2-r}} d x\right)^{\frac{2-r}{r}}
$$




$$
\left(\int_{U}|f|^{r^{*}} d x\right)^{\frac{2}{r^{*}}} \leq C\left(\int_{U} K(\xi)|\nabla f|^{2} d x\right)\left(\int_{U} 1+H(\xi)^{\frac{a r}{(2-a)(2-r)}} d x\right)^{\frac{2-r}{r}} .
$$

Taking $r=2-a$ in (2.31) one obtains (2.29).

When the Degree Condition (2.28) holds, one has $2 \leq(2-a)^{*}$, hence $(2.30)$ follows by (2.29) and Holder inequality.

Notes on Notations: We will drop the constants' indices. The values of $C, C_{1}, C_{2}$, etc., may vary from line to line unless mentioned otherwise. Also, we use different notations for partial derivatives. For example, $\frac{\partial p}{\partial t}=\partial_{t} p=p_{t}$.

\section{Bounds FOR The Solutions}

Our aim is to study the equation (2.7) for pressure of slightly compressible fluids in a bounded domain in a porous media. The fluid flows are subject to some conditions on the boundary.

Let $U$ be a bounded, open, connected subset of $\mathbb{R}^{n}, n=2,3, \ldots$, with $C^{2}$ boundary $\partial U$. (Though we focus on the case $n=3$, the analysis applies to other dimensions.) Let $\partial U=\Gamma_{1} \cup \Gamma_{2}$ with $\Gamma_{1} \cap \Gamma_{2} \neq \emptyset$ and the $(n-1)$-dimensional Lebesgue measure of $\Gamma_{1}$ is nonzero.

In this section, the length $(N+1)$, the exponent vector $\vec{\alpha}$ and the Forchheimer polynomial $g(s, \vec{a}) \in F P(N, \vec{\alpha})$ with $\vec{a} \in R(N)$ are fixed. Throughout, $g(s)=$ $g(s, \vec{a}), K(\xi)=K(\xi, \vec{a})$ and $H(\xi)=H(\xi, \vec{a})$.

Consider the initial boundary value problem for pressure:

$$
\begin{aligned}
& \frac{\partial p}{\partial t}(x, t)=\nabla \cdot(K(|\nabla p(x, t)|) \nabla p(x, t)) \quad \text { on } \quad U \times(0, \infty), \\
& p(x, 0)=p_{0}(x) \quad \text { on } \quad U \\
& \frac{\partial p}{\partial \nu}(x, t)=0 \quad \text { on } \quad \Gamma_{2} \times(0, \infty), \\
& p(x, t)=\psi(x, t) \quad \text { on } \quad \Gamma_{1} \times(0, \infty),
\end{aligned}
$$

where $\nu$ is the outer normal vector on the boundary $\partial U$.

Since our priority is to study the dynamical properties of solutions rather than their regularity, we consider solutions to be classical. The results, however, will be applicable to weak solutions with enough regularities. The boundary data are assumed to have certain regularities accordingly.

For the rest of this section, $p(x, t)$ denotes a solution to the above IBVP (3.1a)(3.1d) with given $p_{0}(x, t)$ and $\psi(x, t)$.

To deal with the non-homogeneous boundary condition, we extend the Dirichlet boundary data $\psi(x, t)$ from $\Gamma_{1}$ to the whole domain $U$. Let $\Psi(x, t)$ be such an extension. For the existence and estimates of $\Psi(x, t)$, see Remark 3.18 below.

Let $\bar{p}=p-\Psi$, then $\bar{p}$ satisfies

$$
\begin{gathered}
\frac{\partial \bar{p}}{\partial t}=\nabla \cdot(K(|\nabla p|) \nabla p)-\Psi_{t} \quad \text { on } U \times(0, \infty), \\
\bar{p}=0 \quad \text { on } \quad \Gamma_{1} \times(0, \infty) .
\end{gathered}
$$

We will derive a priori estimates for solutions $p(x, t)$. Henceforward all constants $C, C_{1}, C_{2}, \ldots$ in this section depend only on parameters $N, \alpha_{N}, \chi(\vec{a})$, the spatial dimension $n$, and the domain $U$.

We denote $H(x, t)=H[p](x, t)=H(|\nabla p(x, t)|, \vec{a})$, see Definition 2.2 . 
We start with a basic differential inequality for the $L^{2}$-norm of $\bar{p}$.

Lemma 3.1. One has

$$
\frac{1}{2} \frac{d}{d t} \int_{U} \bar{p}^{2}(x, t) d x \leq-C \int_{U} H(x, t) d x+C G_{1}(t),
$$

where

$$
G_{1}(t)=\int_{U}|\nabla \Psi(x, t)|^{2} d x+\left(\int_{U}\left|\Psi_{t}(x, t)\right|^{r_{0}} d x\right)^{\frac{2-a}{r_{0}(1-a)}}+\left(\int_{U}\left|\Psi_{t}(x, t)\right|^{r_{0}} d x\right)^{\frac{1}{r_{0}}},
$$

with $r_{0}$ denoting the conjugate exponent of $(2-a)^{*}=n(2-a) /(n-(2-a))$, thus explicitly having the value

$$
r_{0}=\frac{n(2-a)}{(2-a)(n+1)-n}=\frac{n\left(2+\alpha_{N}\right)}{n+2+\alpha_{N}} .
$$

Proof. Multiplying the equation (3.2) by $\bar{p}$ and integrating over $U$, one obtains

$$
\frac{1}{2} \frac{d}{d t} \int_{U} \bar{p}^{2} d x=-\int_{U} K(|\nabla p|) \nabla p \cdot \nabla \bar{p} d x+\int_{\Gamma_{1} \cup \Gamma_{2}} K(|\nabla p|)(\nabla p \cdot \nu) \bar{p} d \sigma-\int_{U} \Psi_{t} \bar{p} d x
$$

Because of the boundary conditions (3.1c) and (3.3) on $p$ and $\bar{p}$, the integrals over the boundaries vanish. Hence

$$
\frac{1}{2} \frac{d}{d t} \int_{U} \bar{p}^{2} d x=-\int_{U} K(|\nabla p|)|\nabla p|^{2} d x+\int_{U} K(|\nabla p|) \nabla p \cdot \nabla \Psi d x-\int_{U} \Psi_{t} \bar{p} d x
$$

First, thanks to relation (2.23) the first integral on the RHS of (3.7) satisfies

$$
-\int_{U} K(|\nabla p|)|\nabla p|^{2} d x \leq-C \int_{U} H(x, t) d x .
$$

We estimate the second integral on the RHS of (3.7) by Holder inequality and the use of relation (2.14):

$$
\begin{aligned}
\left|\int_{U} K(|\nabla p|) \nabla p \cdot \nabla \Psi d x\right| & \leq\left(\int _ { U } ( K ( | \nabla p | ) | \nabla p | ^ { 2 } d x ) ^ { \frac { 1 } { 2 } } \left(\int_{U}\left(K(|\nabla p|)|\nabla \Psi|^{2} d x\right)^{\frac{1}{2}}\right.\right. \\
& \leq C\left(\int_{U} H(x, t) d x\right)^{\frac{1}{2}}\left(\int_{U}|\nabla \Psi|^{2} d x\right)^{\frac{1}{2}} .
\end{aligned}
$$

By Young's inequality, this leads to

$$
\left|\int_{U} K(|\nabla p|) \nabla p \cdot \nabla \Psi d x\right| \leq \varepsilon \int_{U} H(x, t) d x+C \int_{U}|\nabla \Psi|^{2} d x .
$$

For the third integral on the RHS of (3.7), let $b=a /(2-a)$ and $r=r_{0}$. Using Holder inequality, applying Sobolev-Poincare inequality (2.29) with $f=\bar{p}$ and 
$\xi=|\nabla p|$, and using relation $(2.23)$, one obtains

$$
\begin{aligned}
& \int_{U}\left|\Psi_{t} \bar{p}\right| d x \leq\left(\int_{U}\left|\Psi_{t}\right|^{r} d x\right)^{1 / r}\left(\int_{U}|\bar{p}|^{(2-a) *} d x\right)^{1 /(2-a)^{*}} \\
& \leq C\left(\int_{U}\left|\Psi_{t}\right|^{r} d x\right)^{1 / r}\left(\int_{U} K(|\nabla p|)|\nabla \bar{p}|^{2} d x\right)^{\frac{1}{2}}\left(\int 1+K(|\nabla p|)|\nabla p|^{2} d x\right)^{\frac{b}{2}} \\
& \leq C\left(\int_{U}\left|\Psi_{t}\right|^{r} d x\right)^{1 / r}\left(\int_{U} H(x, t)+|\nabla \Psi|^{2} d x\right)^{\frac{1}{2}}\left(\int 1+H(x, t) d x\right)^{\frac{b}{2}} \\
& \leq C\left(\int_{U}\left|\Psi_{t}\right|^{r} d x\right)^{1 / r}\left(\left(\int_{U} H(x, t) d x\right)^{b+1}+\int_{U} H(x, t) d x+\int|\nabla \Psi|^{2} d x\right. \\
& \left.\quad+\int|\nabla \Psi|^{2} d x\left(\int H(x, t) d x\right)^{b}\right)^{\frac{1}{2}} \\
& \leq C\left(\int_{U}\left|\Psi_{t}\right|^{r} d x\right)^{1 / r}\left(1+\left(\int_{U} H(x, t) d x\right)^{b+1}+\left(\int|\nabla \Psi|^{2} d x\right)^{b+1}\right)^{\frac{1}{2}} \\
& \leq C\left(\int_{U}\left|\Psi_{t}\right|^{r} d x\right)^{1 / r}+C\left(\int_{U}\left|\Psi_{t}\right|^{r} d x\right)^{1 / r}\left(\left(\int_{U} H(x, t) d x\right)^{\frac{1}{2-a}}+\left(\int|\nabla \Psi|^{2} d x\right)^{\frac{1}{2-a}}\right) .
\end{aligned}
$$

Applying Young inequality yields

$$
\begin{aligned}
\int_{U}\left|\Psi_{t} \bar{p}\right| d x \leq & \varepsilon \int_{U} H(x, t) d x+C \int_{U}|\nabla \Psi|^{2} d x+C\left(\int_{U}\left|\Psi_{t}\right|^{r} d x\right)^{\frac{1}{r}} \\
& +C\left(\int_{U}\left|\Psi_{t}\right|^{r} d x\right)^{\frac{2-a}{r(1-a)}} .
\end{aligned}
$$

Summing up the above estimates with sufficiently small $\varepsilon$, one obtains

$$
\begin{aligned}
\frac{1}{2} \frac{d}{d t} \int_{U} \bar{p}^{2} d x \leq & -C \int_{U} H(x, t) d x+C \int_{U}|\nabla \Psi|^{2} d x \\
& \left.+C\left(\int_{U}\left|\Psi_{t}\right|^{r} d x\right)^{1 / r}+C \int_{U}\left|\Psi_{t}\right|^{r} d x\right)^{\frac{2-a}{r(1-a)}} .
\end{aligned}
$$

This proves (3.4).

One then obtains the first $L^{2}$-estimates for $\bar{p}$.

Corollary 3.2. One has for $t \geq 0$ that

$$
\int_{U} \bar{p}^{2}(x, t) d x \leq \int_{U} \bar{p}^{2}(x, 0) d x+C \Lambda_{1}(t)
$$

where

$$
\Lambda_{1}(t)=\int_{0}^{t} G_{1}(\tau) d \tau
$$

In the case $\operatorname{deg}(g) \leq 4 /(n-2)$ one has

$$
\int_{U} \bar{p}^{2}(x, t) d x \leq \int_{U} \bar{p}^{2}(x, 0) d x+C \Lambda_{2}(t)
$$

where

$$
\Lambda_{2}(t)=1+\operatorname{Env}\left(G_{1}\right)^{\frac{2}{2-a}}(t),
$$

with $\operatorname{Env}\left(G_{1}\right)(t)$ being a continuous, increasing envelop of the function $G_{1}(t)$ (see Definition A.1). 
Proof. The first inequality (3.9) results from integrating Ineq. (3.4) in time and dropping the first term on its RHS.

To prove the second inequality (3.11), one first observes on one hand that

$$
\int_{U}|\nabla \bar{p}|^{2-a} d x \leq C \int_{U} H(x, t) d x+C \int_{U}|\nabla \Psi|^{2-a} d x+C .
$$

On another hand, the condition on the degree of the polynomial $g$ infers that $2 \leq(2-a)^{*}$. Thus one has by Poincare-Sobolev inequality:

$$
\left(\int_{U} \bar{p}^{2} d x\right)^{\frac{2-a}{2}} \leq C \int_{U}|\nabla \bar{p}|^{2-a} d x
$$

Therefore (3.4), (3.13), and (3.14) give

$$
\frac{1}{2} \frac{d}{d t} \int_{U} \bar{p}^{2} d x \leq-C\left(\int_{U} \bar{p}^{2} d x\right)^{\frac{2-a}{2}}+C G_{1}(t)+C \int_{U}|\nabla \Psi|^{2-a} d x+C .
$$

Since the integral $\int_{U}|\nabla \Psi|^{2-a} d x$ is already present in $G_{1}(t)$, one can adjust the constant $C$ to have

$$
\frac{1}{2} \frac{d}{d t} \int_{U} \bar{p}^{2} d x \leq-C\left(\int_{U} \bar{p}^{2} d x\right)^{\frac{2-a}{2}}+C G_{1}(t)+C .
$$

Applying Lemma A.2 in the Appendix with $y(t)=\int_{U} \bar{p}(x, t)^{2} d x, f(t)=C(1+$ $\left.G_{1}(t)\right)$ and $\alpha=(2-a) / 2$ yields $(3.11)$.

Note that the $L^{2}$-estimate for $p$ easily follows by using

$$
\int_{U} p^{2} d x \leq 2 \int_{U} \bar{p}^{2} d x+2 \int_{U} \Psi^{2} d x
$$

We will not explicate more on this.

Next, we find estimates for $\nabla p$ by using the function $H(x, t)$.

Lemma 3.3. For any $\varepsilon>0$, one has

$$
\frac{d}{d t} \int_{U} H(x, t) d x+\int_{U} \bar{p}_{t}^{2}(x, t) d x \leq \varepsilon \int_{U} H(x, t) d x+C_{\varepsilon} G_{2}(t),
$$

where $C_{\varepsilon}$ is positive and

$$
G_{2}(t)=\int_{U}\left|\nabla \Psi_{t}(x, t)\right|^{2} d x+\int_{U}\left|\Psi_{t}(x, t)\right|^{2} d x
$$

Consequently, one has

$$
\frac{d}{d t}\left[\int_{U} H(x, t)+\bar{p}^{2}(x, t) d x\right]+\int_{U} \bar{p}_{t}^{2}(x, t) d x \leq-C \int_{U} H(x, t) d x+C G_{3}(t)
$$

where

$$
G_{3}(t)=G_{1}(t)+G_{2}(t) .
$$


Proof. Multiplying (3.2) by $\partial \bar{p} / \partial t$, integrating over $U$ and using the boundary conditions of $p$ and $\bar{p}$, one obtains

$$
\begin{aligned}
& \int_{U}\left(\frac{\partial \bar{p}}{\partial t}\right)^{2} d x=-\int_{U} K(|\nabla p|) \nabla p \cdot \frac{\partial}{\partial t}(\nabla \bar{p}) d x+\int_{U} \Psi_{t} \frac{\partial \bar{p}}{\partial t} d x \\
& =-\int_{U} K(|\nabla p|) \nabla p \cdot \frac{\partial}{\partial t}(\nabla p) d x+\int_{U} K(|\nabla p|) \nabla p \cdot \nabla \Psi_{t} d x-\int_{U} \Psi_{t} \frac{\partial \bar{p}}{\partial t} d x \\
& =-\frac{1}{2} \int_{U} \frac{\partial}{\partial t} H(x, t) d x+\int_{U} K(|\nabla p|) \nabla p \cdot \nabla \Psi_{t} d x-\int_{U} \Psi_{t} \frac{\partial \bar{p}}{\partial t} .
\end{aligned}
$$

Hence

$$
\int_{U}\left(\frac{\partial \bar{p}}{\partial t}\right)^{2} d x+\frac{1}{2} \frac{d}{d t} \int_{U} H(x, t) d x=\int_{U} K(|\nabla p|) \nabla p \cdot \nabla \Psi_{t} d x-\int_{U} \Psi_{t} \frac{\partial \bar{p}}{\partial t}
$$

Same as the estimate (3.8), one has

$$
\left|\int_{U} K(|\nabla p|) \nabla p \cdot \nabla \Psi_{t} d x\right| \leq \varepsilon \int_{U} H(x, t) d x+C \int_{U}\left|\nabla \Psi_{t}\right|^{2} d x
$$

For the last integral of (3.21) one uses Cauchy inequality:

$$
\left|\int_{U} \Psi_{t} \frac{\partial \bar{p}}{\partial t}\right| \leq \frac{1}{2} \int_{U}\left(\frac{\partial \bar{p}}{\partial t}\right)^{2} d x+\frac{1}{2} \int_{U} \Psi_{t}^{2} d x
$$

Combining (3.21), (3.22) and (3.23), one obtains (3.17).

Adding (3.4) to (3.17) with sufficiently small $\varepsilon$ yields

$$
\frac{d}{d t}\left[\int_{U} H(x, t)+\bar{p}^{2} d x\right]+\int_{U}\left(\frac{\partial \bar{p}}{\partial t}\right)^{2} d x \leq-C \int_{U} H(x, t) d x+C G_{1}(t)+C G_{2}(t) .
$$

Therefore (3.19) follows.

Remark 3.4. The estimates in Lemma 3.1, Corollary 3.2 and Lemma 3.3 can be improved slightly by replacing $G_{1}(t)$ and $G_{2}(t)$ by the following $\bar{G}_{1}(t)$ and $\bar{G}_{2}(t)$ respectively:

$$
\begin{aligned}
\bar{G}_{1}(t)= & \int_{U}|\nabla \Psi(x, t)|^{2-a} d x+\left(\int_{U}|\nabla \Psi(x, t)|^{2-a} d x\right)^{\frac{1}{2-a}} \\
& +\left(\int_{U}\left|\Psi_{t}(x, t)\right|^{r_{0}} d x\right)^{\frac{2-a}{r_{0}(1-a)}}+\left(\int_{U}\left|\Psi_{t}(x, t)\right|^{r_{0}} d x\right)^{1 / r_{0}}, \\
\bar{G}_{2}(t)= & \int_{U}\left|\nabla \Psi_{t}\right|^{2-a} d x+\left(\int_{U}\left|\nabla \Psi_{t}\right|^{2-a} d x\right)^{\frac{1}{2-a}}+\int_{U} \Psi_{t}^{2} d x .
\end{aligned}
$$


For the proof, one needs to re-estimate (3.8) and (3.22). For instance, one can estimate $\left|\int_{U} K(|\nabla p|) \nabla p \cdot \nabla \Psi d x\right|$ in $(3.8)$ as

$$
\begin{aligned}
& \left|\int_{U} K(|\nabla p|) \nabla p \cdot \nabla \Psi d x\right| \leq C \int_{U}\left(|\nabla p|^{1-a}+1\right)|\nabla \Psi| d x \\
& \leq C\left(\int_{U}|\nabla p|^{2-a}+1 d x\right)^{\frac{1-a}{2-a}}\left(\int_{U}|\nabla \Psi|^{2-a} d x\right)^{\frac{1}{2-a}} \\
& \leq C\left(\int_{U} H(x, t)+1 d x\right)^{\frac{1-a}{2-a}}\left(\int_{U}|\nabla \Psi|^{2-a} d x\right)^{\frac{1}{2-a}} \\
& \leq C\left(\int_{U} H(x, t) d x\right)^{\frac{1-a}{2-a}}\left(\int_{U}|\nabla \Psi|^{2-a} d x\right)^{\frac{1}{2-a}}+C\left(\int_{U}|\nabla \Psi|^{2-a} d x\right)^{\frac{1}{2-a}} \\
& \leq \varepsilon \int_{U} H(x, t) d x+C \int_{U}|\nabla \Psi|^{2-a} d x+C\left(\int_{U}|\nabla \Psi|^{2-a} d x\right)^{\frac{1}{2-a}}
\end{aligned}
$$

The estimate in (3.22) can be treated similarly.

The first inequality (3.17) of Lemma 3.3 immediately yields the estimate of $H(x, t)$ in terms of its initial values.

Corollary 3.5. Given $\delta>0$, there is $C_{\delta}>0$ such that for all $t \geq 0$ one has

$$
\int_{U} H(x, t) d x \leq e^{\delta t} \int_{U} H(x, 0) d x+C_{\delta} \int_{0}^{t} e^{\delta(t-\tau)} G_{2}(\tau) d \tau,
$$

and consequently,

$$
\int_{U}|\nabla p(x, t)|^{2-a} d x \leq e^{\delta t} \int_{U}|\nabla p(x, 0)|^{2-a} d x+C+C_{\delta} \int_{0}^{t} e^{\delta(t-\tau)} G_{2}(\tau) d \tau
$$

Proof. The estimate (3.27) is obtained by letting $\varepsilon=\delta$ and integrating (3.17) in time. Ineq. (3.28) follows by using the relation (2.25).

Remark 3.6. The above estimate for $\int_{U}|\nabla p|^{2-a} d x$ is a direct consequence of the estimate for $\int_{U} H(x, t) d x$ and the relation $(2.25)$. Therefore we will not repeat this derivation in the future.

The estimates in Corollary 3.5 with an exponential growth in time are not appropriate to the study of the asymptotic stability of the solutions. However, they can be improved when combined with the estimate of $\int_{U} \bar{p}^{2}(x, t) d x$.

Corollary 3.7. For $t \geq 0$, one has

$$
\begin{aligned}
\int_{U} H(x, t) d x \leq & e^{-C_{1} t} \int_{U} H(x, 0) d x+C \int_{U} \bar{p}^{2}(x, 0) d x \\
& +C \int_{0}^{t} e^{-C_{1}(t-\tau)}\left(\Lambda_{1}(\tau)+G_{3}(\tau)\right) d \tau .
\end{aligned}
$$

In case $\operatorname{deg}(g) \leq 4 /(n-2)$, one has for $t \geq 0$ that

$$
\begin{aligned}
\int_{U} H(x, t) d x \leq & e^{-C_{1} t} \int_{U} H(x, 0) d x+C \int_{U} \bar{p}^{2}(x, 0) d x \\
& +C \int_{0}^{t} e^{-C_{1}(t-\tau)}\left(\Lambda_{2}(\tau)+G_{3}(\tau)\right) d \tau .
\end{aligned}
$$


Proof. From (3.19), one has

$$
\frac{d}{d t} \int_{U} H(x, t)+C \int_{U} \bar{p}_{t} \bar{p} d x+\int_{U} \bar{p}_{t}^{2} d x \leq-C_{1} \int_{U} H(x, t) d x+C G_{3}(t) .
$$

Applying Cauchy inequality to the term $\int_{U} \overline{p p}_{t} d x$ and using the estimate (3.9) one obtains

$$
\begin{aligned}
& \frac{d}{d t} \int_{U} H(x, t) d x+\frac{1}{2} \int_{U} \bar{p}_{t}^{2} d x \leq-C_{1} \int_{U} H(x, t) d x+C \int_{U} \bar{p}^{2} d x+C G_{3}(t) \\
& \leq-C_{1} \int_{U} H(x, t) d x+C \int_{U} \bar{p}^{2}(x, 0) d x+C \Lambda_{1}(t)+C G_{3}(t) .
\end{aligned}
$$

Neglecting the second integral on the LHS and using Gronwall's inequality one derives

$$
\begin{aligned}
\int_{U} H(x, t) d x \leq & e^{-C_{1} t} \int_{U} H(x, 0) d x \\
& +\int_{0}^{t} e^{-C_{1}(t-\tau)}\left(\int_{U} \bar{p}^{2}(x, 0) d x+C \Lambda_{1}(\tau)+C G_{3}(\tau)\right) d \tau
\end{aligned}
$$

thus proving (3.29).

When $\operatorname{deg}(g) \leq 4 /(n-2)$, using estimate (3.11) instead of (3.9) in the above proof one obtains (3.30).

To take advantage of the dissipation term on the RHS of (3.19), one needs to compare $\int_{U} H d x$ and $\int_{U} \bar{p}^{2} d x$. Hence the following weighted Poincare inequality is needed.

Lemma 3.8. Suppose $\operatorname{deg}(g) \leq \frac{4}{n-2}$. Then

$$
\begin{aligned}
& \int_{U} \bar{p}^{2}(x, t) d x \leq C h(t)\left(\int_{U} H(x, t) d x+\int_{U}|\nabla \Psi(x, t)|^{2} d x\right) \\
& \left(\int_{U} \bar{p}^{2}(x, t) d x\right)^{\frac{2-a}{2}} \leq C\left(1+\int_{U} H(x, t) d x\right)+C \int_{U}|\nabla \Psi(x, t)|^{2} d x
\end{aligned}
$$

where

$$
h(t)=\left(1+\int_{U} H(x, t) d x\right)^{\frac{a}{2-a}} .
$$

Proof. First, one applies (2.30) with $f=\bar{p}$ and $\xi=|\nabla p|$ to obtain

$$
\begin{aligned}
\int_{U} \bar{p}^{2} d x & \leq C h(t)\left(\int_{U} K(|\nabla p|)|\nabla \bar{p}|^{2} d x\right) \\
& \leq C h(t)\left(\int_{U} K(|\nabla p|)\left(|\nabla p|^{2}+|\nabla \Psi|^{2}\right) d x\right) .
\end{aligned}
$$

Hence (3.31) follows. 
One derives from (3.31) and Young inequality:

$$
\begin{aligned}
& \int_{U} \bar{p}^{2} d x \leq C\left(1+\int_{U} H(x, t) d x\right)^{1+\frac{a}{2-a}}+C\left(1+\int_{U} H(x, t) d x\right)^{\frac{a}{2-a}} \int_{U}|\nabla \Psi|^{2} d x \\
& \leq C\left(1+\int_{U} H(x, t) d x\right)^{\frac{2}{2-a}}+C\left(1+\int_{U} H(x, t) d x\right)^{\frac{a}{2-a} \cdot \frac{2}{a}}+\left(\int_{U}|\nabla \Psi|^{2} d x\right)^{\frac{2}{2-a}} \\
& \leq C\left(1+\int_{U} H(x, t) d x\right)^{\frac{2}{2-a}}+\left(\int_{U}|\nabla \Psi|^{2} d x\right)^{\frac{2}{2-a}} \cdot
\end{aligned}
$$

Therefore one obtains (3.32).

Remark 3.9. Concerning the estimate for $H(x, t)$ in Corollary 3.7, thanks to Ineq. (3.32), the second integrals on the RHS of (3.29) and (3.30) can be bounded by

$$
C\left\{1+\left(\int_{U} H(x, 0) d x\right)^{\frac{2}{2-a}}+\left(\int_{U}|\nabla \Psi(x, 0)|^{2} d x\right)^{\frac{2}{2-a}}\right\},
$$

which contains the initial values $H(x, 0)$.

Proposition 3.10. Suppose $\operatorname{deg}(g) \leq \frac{4}{n-2}$. One has the following two estimates

$$
\begin{aligned}
\int_{U} H(x, t)+\bar{p}(x, t)^{2} d x \leq & e^{-C_{1} \int_{0}^{t} h^{-1}(\tau) d \tau}\left(\int_{U} H(x, 0)+\bar{p}(x, 0)^{2} d x\right) \\
& +C \int_{0}^{t} e^{-C_{1} \int_{\tau}^{t} h^{-1}(\theta) d \theta} G_{3}(\tau) d \tau
\end{aligned}
$$

and

$$
\int_{U} H(x, t)+\bar{p}(x, t)^{2} d x \leq \int_{U} H(x, 0)+\bar{p}(x, 0)^{2} d x+C\left(1+\operatorname{Env}\left(G_{3}\right)^{\frac{2}{2-a}}(t)\right)
$$

Proof. From Proposition 3.3:

$$
\begin{aligned}
& \frac{d}{d t}\left[\int_{U} H(x, t)+\bar{p}^{2} d x\right]+\int_{U}\left(\frac{\partial \bar{p}}{\partial t}\right)^{2} d x \\
& \leq-\frac{C}{2} \int_{U} H(x, t) d x-\frac{C}{2} \int_{U} H(x, t) d x+C G_{3}(t) \\
& \leq-\frac{C}{2} \int_{U} H(x, t) d x-\frac{C}{h(t)} \int_{U} \bar{p}^{2} d x+C G_{3}(t)+\int|\nabla \Psi|^{2} d x \\
& \leq-\frac{C}{2} \int_{U} H(x, t) d x-\frac{C}{h(t)} \int_{U} \bar{p}^{2} d x+C G_{3}(t) .
\end{aligned}
$$

Note that $h(t) \geq 1$. Therefore

$$
\begin{aligned}
\frac{d}{d t}\left[\int_{U} H(x, t)+\bar{p}^{2} d x\right] & +\int_{U}\left(\frac{\partial \bar{p}}{\partial t}\right)^{2} d x \\
& \leq-C h^{-1}(t)\left[\int_{U} H(x, t)+\bar{p}^{2} d x\right]+C G_{3}(t)
\end{aligned}
$$

Applying Gronwall's inequality to (3.36) yields (3.34). 
Similarly, by using (3.32):

$$
\begin{aligned}
& \frac{d}{d t}\left[\int_{U} H(x, t)+\bar{p}^{2} d x\right]+\int_{U}\left(\frac{\partial \bar{p}}{\partial t}\right)^{2} d x \\
& \leq-\frac{C}{2} \int_{U} H(x, t) d x+C+\int_{U}|\nabla \Psi|^{2} d x-C\left(\int_{U} \bar{p}^{2} d x\right)^{\frac{2-a}{2}}+C G_{3}(t) \\
& \leq-C\left(1+\int_{U} H(x, t) d x\right)-C\left(\int_{U} \bar{p}^{2} d x\right)^{\frac{2-a}{2}}+C\left(1+G_{3}(t)\right)
\end{aligned}
$$

Since $(2-a) / 2 \leq 1$ and $1+\int_{U} H d x \geq 1$, the above inequality implies

$$
\begin{aligned}
& \frac{d}{d t}\left[1+\int_{U} H(x, t)+\bar{p}^{2} d x\right]+\int_{U}\left(\frac{\partial \bar{p}}{\partial t}\right)^{2} d x \\
& \leq-C\left[1+\int_{U} H(x, t)+\bar{p}^{2} d x\right]^{\frac{2-a}{2}}+C\left(1+G_{3}(t)\right)
\end{aligned}
$$

Then apply Lemma A.2 with $y(t)=1+\int_{U} H(x, t) d x+\int_{U} \bar{p}^{2}(x, t) d x$ to have $y(t) \leq y(0)+C\left(1+\operatorname{Env}\left(G_{3}\right)(t)\right)^{\frac{2}{2-a}}$. Cancelling out numbers 1's from both sides yields (3.35).

Note that in the case when $h(t)$ is unbounded, the estimate (3.35) is more suitable than (3.34).

When the solution has more regularity in the time variable, we derive estimates for $\partial p / \partial t$. As one can see below, this requires more regularity for $\Psi$. We start with a differential inequality for $\partial \bar{p} / \partial t$.

We denote

$$
q(x, t)=p_{t}(x, t) \quad \text { and } \quad \bar{q}(x, t)=\bar{p}_{t}(x, t)=p_{t}(x, t)-\Psi_{t}(x, t)
$$

One has $\left.\bar{q}\right|_{\Gamma_{i}}=0, \nabla \bar{q}=\nabla q-\nabla \Psi_{t}$, and the function $\bar{q}(x, t)$ satisfies the equation

$$
\frac{d \bar{q}}{d t}=\nabla \cdot(K(|\nabla p|) \nabla q)+\nabla \cdot\left(K^{\prime}(|\nabla p|) \frac{\nabla p \cdot \nabla q}{|\nabla p|} \nabla p\right)-\Psi_{t t}(x, t) .
$$

Lemma 3.11. One has for $t>0$ that

$$
\frac{d}{d t} \int_{U} \bar{q}^{2} d x \leq-C \int_{U} K(|\nabla p|)|\nabla \bar{q}|^{2} d x+C \int_{U}\left|\nabla \Psi_{t}\right|^{2} d x+\int_{U}|\bar{q}|\left|\Psi_{t t}\right| d x
$$

Proof. Multiplying Eq. (3.38) by $\bar{q}$, integrating over $U$ and performing integration by parts one gets 


$$
\begin{aligned}
\frac{d}{d t} \int_{U} \bar{q}^{2} d x \\
=-\int_{U} K(|\nabla p|) \nabla q \cdot \nabla \bar{q} d x-\int_{U} K^{\prime}(|\nabla p|) \frac{(\nabla p \cdot \nabla q)(\nabla p \cdot \nabla \bar{q})}{|\nabla p|} d x-\int_{U} \bar{q} \Psi_{t t}(x, t) d x \\
=-\int_{U} K(|\nabla p|)|\nabla q|^{2} d x+\int_{U} K(|\nabla p|) \nabla q \cdot \nabla \Psi_{t} d x \\
\quad-\int_{U} K^{\prime}(|\nabla p|) \frac{(\nabla p \cdot \nabla q)(\nabla p \cdot \nabla q)}{|\nabla p|} d x+\int_{U} K^{\prime}(|\nabla p|) \frac{(\nabla p \cdot \nabla q)\left(\nabla p \cdot \nabla \Psi_{t}\right)}{|\nabla p|} d x \\
\quad-\int_{U} \bar{q} \Psi_{t t}(x, t) d x .
\end{aligned}
$$

By Cauchy-Schwarz inequality and (2.21), one has

$$
\begin{gathered}
K(|\nabla p|)\left|\nabla q \cdot \nabla \Psi_{t}\right| \leq K(|\nabla p|)|\nabla q|\left|\nabla \Psi_{t}\right|, \\
\left|K^{\prime}(|\nabla p|) \frac{(\nabla p \cdot \nabla q)^{2}}{|\nabla p|}\right| \leq\left|K^{\prime}(|\nabla p|)\right||\nabla p||\nabla q|^{2} \leq a K(|\nabla p|)|\nabla q|^{2},
\end{gathered}
$$

and

$$
\begin{aligned}
\left|K^{\prime}(|\nabla p|) \frac{(\nabla p \cdot \nabla q)\left(\nabla p \cdot \nabla \Psi_{t}\right)}{|\nabla p|}\right| & \leq\left|K^{\prime}(|\nabla p|)\right||\nabla p||\nabla q|\left|\nabla \Psi_{t}\right| \\
& \leq a K(|\nabla p|)|\nabla q|\left|\nabla \Psi_{t}\right|,
\end{aligned}
$$

where $a \in[0,1)$ is defined in $(2.10)$. Therefore

$$
\begin{aligned}
\frac{d}{d t} \int_{U} \bar{q}^{2} d x \leq & -(1-a) \int_{U} K(|\nabla p|)|\nabla q|^{2} d x \\
& +(1+a) \int_{U} K(|\nabla p|)|\nabla q|\left|\nabla \Psi_{t}\right| d x+\int_{U}|\bar{q}|\left|\Psi_{t t}\right| d x
\end{aligned}
$$

By Cauchy inequality:

$$
\int_{U} K(|\nabla p|)|\nabla q|\left|\nabla \Psi_{t}\right| d x \leq \varepsilon \int_{U} K(|\nabla p|)|\nabla q|^{2}+C_{\varepsilon} \int_{U}\left|\nabla \Psi_{t}\right|^{2} d x .
$$

Combining (3.40) and (3.41) with sufficiently small $\varepsilon$ one obtains (3.39).

We obtain $L^{2}$-estimates for $\bar{q}$.

Proposition 3.12. One has

$$
\begin{aligned}
& \int_{U} \bar{p}_{t}^{2}(x, t) d x \leq \int_{U} \bar{p}_{t}^{2}(x, 0) d x \\
& +C \int_{0}^{t}\left\{\int_{U}\left|\nabla \Psi_{t}(x, \tau)\right|^{2} d x+h(\tau)\left(\int_{U}\left|\Psi_{t t}(x, \tau)\right|^{r_{0}} d x\right)^{\frac{2}{r_{0}}}\right\} d \tau .
\end{aligned}
$$

In case $\operatorname{deg}(g) \leq \frac{4}{n-2}$, one has

$$
\begin{aligned}
& \int_{U} \bar{p}_{t}^{2}(x, t) d x \leq e^{-C_{1} \int_{0}^{t} \frac{1}{h(\tau)} d \tau} \int_{U} \bar{p}_{t}^{2}(x, 0) d x \\
& +C \int_{0}^{t} e^{-C_{1} \int_{\tau}^{t} \frac{1}{h(\theta)} d \theta}\left(\int_{U}\left|\nabla \Psi_{t}(x, \tau)\right|^{2} d x+h(\tau) \int_{U}\left|\Psi_{t t}(x, \tau)\right|^{2} d x\right) d \tau .
\end{aligned}
$$


Proof. Applying (2.29) with $f=\bar{q}$ and $\xi=|\nabla p|$ to estimate the first integral on the RHS of (3.39), applying Holder then Cauchy inequalities to its last integral, one obtains

$$
\begin{aligned}
\frac{d}{d t} \int_{U} \bar{q}^{2} d x \leq & -C \int_{U} K(|\nabla p|)|\nabla \bar{q}|^{2} d x+C \int_{U}\left|\nabla \Psi_{t}\right|^{2} d x+\int_{U}|\bar{q}|\left|\Psi_{t t}\right| d x \\
\leq & -\frac{C}{h(t)}\left(\int_{U}|\bar{q}|^{(2-a)^{*}} d x\right)^{\frac{2}{(2-a)^{*}}}+C \int_{U}\left|\nabla \Psi_{t}\right|^{2} d x \\
& +\left(\int_{U}|\bar{q}|^{(2-a)^{*}} d x\right)^{\frac{1}{(2-a)^{*}}}\left(\int_{U}\left|\Psi_{t t}\right|^{r_{0}} d x\right)^{\frac{1}{r_{0}}} \\
\leq & -\frac{C}{h(t)}\left(\int_{U}|\bar{q}|^{(2-a)^{*}} d x\right)^{\frac{2}{(2-a)^{*}}}+C \int_{U}\left|\nabla \Psi_{t}\right|^{2} d x \\
& +\frac{C \varepsilon}{h(t)}\left(\int_{U}|\bar{q}|^{(2-a)^{*}} d x\right)^{\frac{2}{(2-a)^{*}}}+C_{\varepsilon} h(t)\left(\int_{U}\left|\Psi_{t t}\right|^{r_{0}} d x\right)^{\frac{2}{r_{0}}}
\end{aligned}
$$

With sufficient small $\varepsilon$ it follows that

(3.44)

$\frac{d}{d t} \int_{U} \bar{q}^{2} d x \leq-\frac{C}{h(t)}\left(\int_{U}|\bar{q}|^{(2-a)^{*}} d x\right)^{\frac{2}{(2-a)^{*}}+C} \int_{U}\left|\nabla \Psi_{t}\right|^{2} d x+C h(t)\left(\int_{U}\left|\Psi_{t t}\right|^{r_{0}} d x\right)^{\frac{2}{r_{0}}}$.

Neglecting the first term on the RHS and integrating in time yield (3.42).

Now when $\operatorname{deg}(g) \leq \frac{4}{n-2}$, one has $2 \leq(2-a)^{*}$. By using Holder inequality in (3.44), one asserts

$$
\frac{d}{d t} \int_{U} \bar{q}^{2} d x \leq-\frac{C}{h(t)} \int_{U} \bar{q}^{2} d x+C \int_{U}\left|\nabla \Psi_{t}\right|^{2} d x+C h(t) \int_{U}\left|\Psi_{t t}\right|^{2} d x
$$

Then apply Gronwall's inequality to obtain (3.43).

Now one relates the estimate of $\bar{p}_{t}$ with those of $H(x, t)$ and $\bar{p}$.

Lemma 3.13. One has for all $t \geq 0$ that

$$
\frac{1}{2} \frac{d}{d t}\left[\int_{U} H(x, t)+\bar{p}_{t}^{2}(x, t)+\bar{p}^{2}(x, t) d x\right] \leq-C_{1}\left[\int_{U} H(x, t)+\bar{p}_{t}^{2} d x\right]+C G_{4}(t),
$$

where

$$
G_{4}(t)=G_{3}(t)+\int_{U} \Psi_{t t}^{2}(x, t) d x .
$$

Proof. Applying Cauchy inequality to the last integral of (3.39) yields

$$
\frac{d}{d t} \int_{U} \bar{q}^{2} d x \leq-C \int_{U} K(|\nabla p|)|\nabla q|^{2} d x+C \int_{U}\left|\nabla \Psi_{t}\right|^{2} d x+\varepsilon \int_{U} \bar{q}^{2}+C \int_{U} \Psi_{t t}^{2} d x .
$$

Summing up (3.19) and (3.47), one obtains

$$
\begin{aligned}
& \frac{1}{2} \frac{d}{d t}\left[\int_{U} H(x, t)+\bar{p}_{t}^{2}+\bar{p}^{2} d x\right] \leq-C_{1} \int_{U} H(x, t) d x-C \int_{U} \bar{p}_{t}^{2} d x \\
& -C_{1} \int_{U} K(|\nabla p|)|\nabla q|^{2} d x+\varepsilon \int_{U} \bar{p}_{t}^{2} d x+C G_{3}(t)+C \int_{U}\left|\nabla \Psi_{t}\right|^{2} d x+C \int_{U} \Psi_{t t}^{2} d x .
\end{aligned}
$$

By selecting $\varepsilon$ sufficiently small, one obtains (3.45). 
We estimate the Lebesgue norms of the space-time derivatives of $p$.

Corollary 3.14. One has for $t \geq 0$,

$$
\begin{aligned}
& \int_{U} H(x, t) d x+\int_{U} \bar{p}_{t}^{2}(x, t) d x \leq e^{-C_{1} t}\left(\int_{U} H(x, 0) d x+\int_{U} \bar{p}_{t}^{2}(x, 0) d x\right) \\
& +C \int_{U} \bar{p}^{2}(x, 0) d x+C \int_{0}^{t} e^{-C_{1}(t-\tau)}\left(\Lambda_{1}(\tau)+G_{4}(\tau)\right) d \tau
\end{aligned}
$$

Proof. The proof is similar to Corollary 3.7. In (3.45), one applies Cauchy inequality to the term

$$
\frac{d}{d t} \int_{U} \bar{p}^{2} d x=2 \int_{U} \bar{p}_{t} \bar{p} d x
$$

and uses the estimate (3.9):

$$
\begin{aligned}
& \frac{1}{2} \frac{d}{d t}\left[\int_{U} H(x, t)+\bar{p}_{t}^{2} d x\right] \\
& \leq-C\left[\int_{U} H(x, t)+\bar{p}_{t}^{2} d x\right]+C \int_{U} \bar{p}(x, t)^{2} d x+C G_{4}(t) \\
& \leq-C\left[\int_{U} H(x, t)+\bar{p}_{t}^{2} d x\right]+C \int_{U} \bar{p}(x, 0)^{2} d x+C \Lambda_{1}(t)+C G_{4}(t) .
\end{aligned}
$$

Then (3.48) follows by the Gronwall inequality.

Under the Degree Condition (2.28), one can combine the estimate of the derivatives of $p$ above with that of $p$ itself to obtain a stronger result.

Proposition 3.15. Suppose $\operatorname{deg}(g) \leq 4 /(n-2)$. Then

$$
\begin{aligned}
{\left[\int_{U} H(x, t)\right.} & \left.+\bar{p}_{t}^{2}(x, t)+\bar{p}^{2}(x, t) d x\right] \\
\leq & e^{-C_{1} \int_{0}^{t} h^{-1}(\tau) d \tau}\left[\int_{U} H(x, 0)+\bar{p}_{t}^{2}(x, 0)+\bar{p}^{2}(x, 0) d x\right] \\
& +C \int_{0}^{t} e^{-C_{1} \int_{\tau}^{t} h^{-1}(\theta) d \theta} G_{4}(\tau) d \tau
\end{aligned}
$$

Assume, in addition, that

$$
\int_{0}^{t} e^{-C_{1}(t-\tau)}\left(\Lambda_{2}(t)+G_{3}(t)\right) d \tau \leq C_{2} \quad \text { for all } \quad t>0 .
$$

Then there is $d_{0}>0$ depending on the initial data of the solution so that

$$
\begin{aligned}
& {\left[\int_{U} H(x, t)+\bar{p}_{t}^{2}(x, t)+\bar{p}^{2}(x, t) d x\right]} \\
& \leq e^{-d_{0} t}\left[\int_{U} H(x, 0)+\bar{p}_{t}^{2}(x, 0)+\bar{p}^{2}(x, 0) d x\right]+C \int_{0}^{t} e^{-d_{0}(t-\tau)} G_{4}(\tau) d \tau .
\end{aligned}
$$


Proof. One can easily obtain from (3.45) and (3.31) that

$$
\begin{aligned}
& \frac{1}{2} \frac{d}{d t}\left[\int_{U} H(x, t)+\bar{p}_{t}^{2}+\bar{p}^{2} d x\right] \\
& \leq-\frac{C_{1}}{2} \int_{U} H(x, t) d x-\frac{C_{1}}{2} \int_{U} H(x, t) d x-C_{1} \int_{U} \bar{p}_{t}^{2} d x+C G_{4}(t) \\
& \leq-\frac{C_{1}}{2} \int_{U} H(x, t) d x-C h^{-1}(t) \int_{U} \bar{p}^{2} d x+C \int_{U}|\nabla \Psi|^{2} d x-C_{1} \int_{U} \bar{p}_{t}^{2} d x+C G_{4}(t) \\
& \leq-C h^{-1}(t)\left[\int_{U} H(x, t)+\bar{p}_{t}^{2}+\bar{p}^{2} d x\right]+C G_{4}(t) .
\end{aligned}
$$

Then applying Gronwall's inequality yields (3.49).

Under condition (3.50), one observes from estimate (3.30) of Corollary 3.7 that $\int_{U} H(x, t) d x \leq d_{1}$ for all $t \geq 0$. Hence $h(t) \leq d_{2}$ and $h^{-1}(t) \geq d_{3}$ for all $t>0$. Therefore (3.51) follows from (3.49).

Remark 3.16. Condition (3.50) guarantees the Poincare inequality

$$
\int_{U} \bar{p}^{2}(x, t) d x \leq d_{*} \int_{U} K(|\nabla p|)|\nabla \bar{p}|^{2} d x \leq d_{*} \int_{U} H(x, t) d x+d_{*} \int_{U}|\nabla \Psi(x, t)|^{2} d x,
$$

where $d_{*}>0$ is independent of time.

When no condition on $h(t)$ is imposed, one obtains an alternative but simpler estimate than (3.49).

Proposition 3.17. Suppose $\operatorname{deg}(g) \leq \frac{4}{n-2}$. One has

$$
\begin{aligned}
\int_{U} H(x, t)+\bar{p}_{t}^{2}(x, t)+\bar{p}^{2}(x, t) d x \leq & \int_{U} H(x, 0)+\bar{p}_{t}^{2}(x, 0)+\bar{p}^{2}(x, 0) d x \\
& +C\left(1+\operatorname{Env}\left(G_{4}\right)^{\frac{2}{2-a}}(t)\right) .
\end{aligned}
$$

Proof. Similar to the proof of (3.35), but starting from (3.45) instead of (3.19) one has

$$
\begin{aligned}
& \frac{1}{2} \frac{d}{d t}\left[1+\int_{U} H(x, t)+\bar{p}_{t}^{2}+\bar{p}^{2} d x\right] \\
& \leq-C_{1}\left[1+\int_{U} H(x, t)+\bar{p}_{t}^{2}+\bar{p}^{2} d x\right]^{\frac{2-a}{2}}+C\left(1+G_{4}(t)\right) .
\end{aligned}
$$

Then applying Lemma A.2 yields (3.52).

Remark 3.18. The IBVP (3.1a)-(3.1d) is formulated in terms of the boundary profile $\psi(x, t)$. However, in all the results above, the estimates of the solutions depend on a particular extension $\Psi(x, t)$ and its properties. Nonetheless, one can always relate the estimates concerning $\Psi$ in $U$ to those of $\psi$ on $\Gamma_{1}$. For instance, one can use the following harmonic extension $\Psi$ of $\psi$ :

$$
\Delta \Psi=0 \quad \text { on } \quad U \quad \text { and }\left.\quad \Psi\right|_{\Gamma_{1}}=\psi,\left.\quad \frac{\partial \Psi}{\partial \nu}\right|_{\Gamma_{2}}=0 .
$$

We denote such $\Psi$ by $\mathcal{H}(\psi)$. Then we have

$$
\left\|\partial_{t}^{k} \mathcal{H}(\psi)\right\|_{W^{1, s}(U)} \leq C(k, s)\left\|\partial_{t}^{k} \psi\right\|_{W^{1, s}\left(\Gamma_{1}\right)}
$$

for $k=0,1,2$, and $s \geq 1$, c.f. [14]. 


\section{Dependence on the Boundary Data}

First, we recall from [1] that the solution of IBVP (3.1a)-(3.1d) with a fixed boundary data $\psi(x, t)$ is unique and Lyapunov stable. More precisely, if $p_{1}$ and $p_{2}$ are two such solutions, then for $t \geq 0$ one has

$$
\left\|p_{1}(\cdot, t)-p_{2}(\cdot, t)\right\|_{L^{2}(U)} \leq\left\|p_{1}(\cdot, 0)-p_{2}(\cdot, 0)\right\|_{L^{2}(U)} .
$$

We now turn to studying the IBVP (3.1a)-(3.1d) with varying boundary data.

4.1. Spatial homogeneous boundary data and their perturbations. In this subsection, qualitative behavior of the solutions are studied using the results obtained in sections 2 and 3 . The simplest consideration is the stability, with respect to perturbations of the boundary data, of the homogeneous solutions in time and space, i.e., $p(x, t)=$ const., or, $|\nabla p(x, t)|=p_{t}(x, t)=0$. In this case the initial and boundary data are also constants. More generally, we consider the boundary data which depends on time only, i.e., homogeneous in the spatial variables. This boundary condition models processes on the boundary, when the domain adjacent to $\Gamma_{1}$ possesses infinite conductivity. Perturbations of those data and their corresponding solutions are studied.

First, we quickly obtain the Lyapunov stability for homogeneous solutions.

Proposition 4.1. Suppose $\operatorname{deg}(g) \leq 4 /(n-2)$. Assume that $\psi(x, t)=A+\phi(x, t)$ on $\Gamma_{1}$ with $\phi(x, t)$ satisfying

$$
\sup _{[0, \infty)}\|\phi(\cdot, t)\|_{W^{1,2}\left(\Gamma_{1}\right)}, \sup _{[0, \infty)}\left\|\phi_{t}(\cdot, t)\right\|_{W^{1,2}\left(\Gamma_{1}\right)}, \sup _{[0, \infty)}\left\|\phi_{t t}(\cdot, t)\right\|_{W^{1,2}\left(\Gamma_{1}\right)}<\infty .
$$

Let $p(x, t)$ be the corresponding solution to IBVP (3.1a)-(3.1d) and $z(x, t)=$ $p(x, t)-A$. Then

$$
\begin{aligned}
& \sup _{[0, \infty)} \int_{U}|\nabla z(x, t)|^{2-a}+z_{t}^{2}(x, t)+z^{2}(x, t) d x \\
\leq & C \int_{U}|\nabla z(x, 0)|^{2-a}+z_{t}^{2}(x, 0)+z^{2}(x, 0) d x+C\left\{\sup _{[0, \infty)}\|\phi(\cdot, t)\|_{W^{1,2}\left(\Gamma_{1}\right)}^{2}\right. \\
+ & \left.\sup _{[0, \infty)}\left\|\phi_{t}(\cdot, t)\right\|_{W^{1,2}\left(\Gamma_{1}\right)}+\sup _{[0, \infty)}\left\|\phi_{t}(\cdot, t)\right\|_{W^{1,2}\left(\Gamma_{1}\right)}^{\frac{2-a}{1-a}}+\sup _{[0, \infty)}\left\|\phi_{t t}(\cdot, t)\right\|_{W^{1,2}\left(\Gamma_{1}\right)}^{2}\right\} .
\end{aligned}
$$

Proof. Let $\Phi(x, t)$ be the harmonic extension of $\phi(x, t)$ as defined in Remark 3.18. Let $\Psi(x, t)=A+\Phi(x, t)$. Then one has $\bar{p}=p-\Psi=z-\Phi$. Note that $\nabla \Psi=\nabla \Phi$ and $\Psi_{t}=\Phi_{t}$.

Let $D_{*}=1+\left(\sup _{[0, \infty)} G_{1}(t)\right)^{\frac{2}{2-a}}+\sup _{[0, \infty)} G_{2}(t)$. Using the estimate in Remark 3.18 and condition (4.2), one has $D_{*}<\infty$ and

$$
G_{1}(t), G_{2}(t), G_{3}(t), \Lambda_{2}(t) \leq C D_{*},
$$

hence $h(t) \leq C D_{*}^{b}$. By (3.51) in Proposition 3.15, one has 


$$
\begin{aligned}
{\left[\int_{U} H(x, t)\right.} & \left.+\bar{p}_{t}^{2}(x, t)+\bar{p}^{2}(x, t) d x\right] \\
\leq & e^{-C_{1} \int_{0}^{t} h^{-1}(\tau) d \tau}\left[\int_{U} H(x, 0)+\bar{p}_{t}^{2}(x, 0)+\bar{p}^{2}(x, 0) d x\right] \\
& +\int_{0}^{t} e^{-C_{1} \int_{\tau}^{t} h^{-1}(\theta) d \theta} G_{4}(\tau) d \tau \\
\leq & {\left[\int_{U} H(x, 0)+\bar{p}_{t}^{2}(x, 0)+\bar{p}^{2}(x, 0) d x\right]+\int_{0}^{t} e^{-C_{1} D_{*}^{-b}(t-\tau)} G_{4} d \tau } \\
\leq & {\left[\int_{U} H(x, 0)+\bar{p}_{t}^{2}(x, 0)+\bar{p}^{2}(x, 0) d x\right]+C D_{*}^{b} \sup _{[0, \infty)} G_{4}(t) . }
\end{aligned}
$$

Since $\nabla p=\nabla z$ and $p_{t}=z_{t}$, therefore

$$
\begin{aligned}
& {\left[\int_{U}|\nabla z(x, t)|^{2-a}+z_{t}^{2}(x, t)+z^{2}(x, t) d x\right]} \\
& \leq C\left[\int_{U}|\nabla z(x, 0)|^{2-a}+z_{t}^{2}(x, 0)+z^{2}(x, 0) d x\right]+C \sup _{[0, \infty)}\|\Phi(\cdot, t)\|_{L^{2}}^{2} \\
& +C \sup _{[0, \infty)}\left\|\Phi_{t}(\cdot, t)\right\|_{L^{2}}^{2}+C D_{*}^{b} \sup _{[0, \infty)} G_{4}(t) \\
& \leq C\left[\int_{U}|\nabla z(x, 0)|^{2-a}+z_{t}^{2}(x, 0)+z^{2}(x, 0) d x\right]+C \sup _{[0, \infty)}\|\Phi(\cdot, t)\|_{L^{2}}^{2} \\
& +C \sup _{[0, \infty)}\left\|\Phi_{t}(\cdot, t)\right\|_{L^{2}}^{2}+C D_{*}^{b} \sup _{[0, \infty)}\left\{\left\|\Phi_{t}(\cdot, t)\right\|_{L^{2}}^{2}+\left\|\Phi_{t}(\cdot, t)\right\|_{L^{r_{0}}}\right. \\
& \left.+\left\|\Phi_{t}(\cdot, t)\right\|_{L^{r_{0}}}^{\frac{2-a}{1-a}}+\left\|\Phi_{t t}(\cdot, t)\right\|_{L^{2}}^{2}+\|\nabla \Phi(\cdot, t)\|_{L^{2}}^{2}+\left\|\nabla \Phi_{t}(\cdot, t)\right\|_{L^{2}}^{2}\right\} .
\end{aligned}
$$

By Holder inequality with $r_{0} \leq 2$ and Young inequality with $1<2 \leq \frac{2-a}{1-a}$ one has

$$
\left\|\Phi_{t}(\cdot, t)\right\|_{L^{2}}^{2}+\left\|\Phi_{t}(\cdot, t)\right\|_{L^{r_{0}}}+\left\|\Phi_{t}(\cdot, t)\right\|_{L^{r_{0}}}^{\frac{2-a}{1-a}} \leq C\left(\left\|\Phi_{t}(\cdot, t)\right\|_{L^{2}}+\left\|\Phi_{t}(\cdot, t)\right\|_{L^{2}}^{\frac{2-a}{1-a}}\right) .
$$

Using the estimates of $\Phi$ and its derivatives in Remark 3.18 again, one obtains (4.3).

We now focus on the asymptotic stability. We formulate a result for more general boundary data with some decay at infinity. This decay is expressed in terms of the extension $\Psi(x, t)$.

Proposition 4.2. Suppose $\operatorname{deg}(g) \leq 4 /(n-2)$. Assume that

(a) $\lim _{t \rightarrow \infty}\|\nabla \Psi(\cdot, t)\|_{L^{2}}=0, \quad(b) \lim _{t \rightarrow \infty}\left\|\Psi_{t}(\cdot, t)\right\|_{L^{2}}=0, \quad(c) \lim _{t \rightarrow \infty}\left\|\nabla \Psi_{t}(\cdot, t)\right\|_{L^{2}}=0$.

Then:

(i) The functional

$$
E_{1}(t) \stackrel{\text { def }}{=} \int_{U}\left(H(x, t)+|p(x, t)-\Psi(x, t)|^{2}\right) d x \rightarrow 0, \text { as } t \rightarrow \infty .
$$

(ii) If, in addition, $\|\Psi(\cdot, t)\|_{L^{2}} \rightarrow 0$ as $t \rightarrow \infty$ then

$$
I_{1}(t) \stackrel{\text { def }}{=} \int_{U}\left(|\nabla p(x, t)|^{2-a}+p^{2}(x, t)\right) d x \rightarrow 0, \text { as } t \rightarrow \infty .
$$


Proof. (i) The condition on $\operatorname{deg}(g)$ gives $r_{0} \leq 2$, therefore (b) yields $\left\|\Psi_{t}\right\|_{L^{r_{0}}} \rightarrow 0$. Three conditions (a)-(c) imply that $G_{1}(t), G_{2}(t)$ and $G_{3}(t)$ defined by (3.5), (3.18), and (3.20), respectively, converge to zero as $t \rightarrow \infty$. Therefore $\Lambda_{2}$ in (3.12) is bounded, which implies $\int_{U} H(x, t) d x \leq d_{1}$, by (3.30), where $d_{1}$ depends also on the initial data of the solution. Subsequently, $1 \leq h(t) \leq d_{2}^{-1}$ and hence

$$
d_{2} t \leq S(t) \stackrel{\text { def }}{=} \int_{0}^{t} h^{-1}(\tau) d \tau \leq t \text { and } \lim _{t \rightarrow \infty} S(t)=\infty .
$$

By (3.34) one has

$$
E_{1}(t) \leq e^{-d_{3} t} E_{1}(0)+C e^{-d_{3} S(t)} J_{1}(t),
$$

where $J_{1}(t)=\int_{0}^{t} e^{d_{3} S(\tau)} G_{3}(\tau) d \tau$ with $d_{3}$ depending on the initial data.

In the case $J_{1}(t)$ is bounded, one obviously sees from (4.7) and (4.6) that $E_{1}(t)$ decays exponentially. Otherwise, one applies L'Hôpital's Rule and the fact that $\lim _{t \rightarrow \infty} h(t) G_{3}(t)=0$ to conclude that the second term on the RHS of (4.7) also converges to zero. Therefore one obtains (4.4).

(ii) By virtue of inequalities (2.23) and (4.7) one has

$$
\begin{aligned}
I_{1}(t) & \leq C_{\delta} \int_{U} H(x, t) d x+C \delta^{2-a}+\int_{U} p^{2}(x, t) d x \\
& \leq C_{\delta} E_{1}(t)+C \delta^{2-a}+C_{\delta} \int_{U}|\Psi(x, t)|^{2} d x .
\end{aligned}
$$

Therefore $\lim \sup _{t \rightarrow \infty} I_{1}(t) \leq C \delta^{2-a}$ for all $\delta>0$. Thus $\lim _{t \rightarrow \infty} I_{1}(t)=0$.

Furthermore, if the second derivative $\Psi_{t t}(x, t)$ decays at infinity, then one can also control the $L^{2}$-norm of $p_{t}$.

Proposition 4.3. Suppose $\operatorname{deg}(g) \leq 4 /(n-2)$. Assume in addition to (a)-(c) in Proposition 4.2 that one has

$$
\text { (d) } \lim _{t \rightarrow \infty}\left\|\Psi_{t t}(\cdot, t)\right\|_{L^{2}}=0 .
$$

Then:

(i) The functional

$$
E_{2}(t) \stackrel{\text { def }}{=} E_{1}(t)+\int_{U} p_{t}^{2}(x, t) d x \rightarrow 0, \text { as } t \rightarrow \infty .
$$

(ii) If, in addition, $\lim _{t \rightarrow \infty}\|\Psi(\cdot, t)\|_{L^{2}}=0$ then

$$
I_{2}(t) \stackrel{\text { def }}{=} I_{1}(t)+\int_{U} p_{t}^{2}(x, t) d x \rightarrow 0 \text {, as } t \rightarrow \infty .
$$

Proof. The proof is similar to that of Proposition 4.2 with the use of Proposition 3.15. One has $G_{k}(t) \rightarrow 0$ as $t \rightarrow \infty$ for $k=1,2,3,4$, and $\Lambda_{2}(t) \leq C$. Hence the condition (3.50) is satisfied. The estimate (3.51) gives

$$
\begin{aligned}
E_{2}(t) \leq & \left.C e^{-C t}\left[E_{2}(0)+\int_{U} \Psi_{t}(x, 0)\right)^{2} d x\right] \\
& +C \int_{0}^{t} e^{-C(t-\tau)} G_{4}(\tau) d \tau+C \int_{U} \Psi_{t}^{2}(x, t) d x .
\end{aligned}
$$

The proof now proceeds as in Propositions 4.2. We omit the details. 
The case of spatial homogeneous boundary data and their perturbations is a direct consequence of Propositions 4.2 and 4.3.

Corollary 4.4. Suppose $\operatorname{deg}(g) \leq 4 /(n-2)$. Let $p(x, t)$, be the solution of IBVP (3.1a)-(3.1d) with boundary data $\psi(x, t)=\gamma(t)+\phi(x, t)$, on $\Gamma_{1}$.

(i) Assume

$$
\lim _{t \rightarrow \infty}\left(\left|\gamma^{\prime}(t)\right|+\|\phi(\cdot, t)\|_{W^{1,2}\left(\Gamma_{1}\right)}+\left\|\phi_{t}(\cdot, t)\right\|_{W^{1,2}\left(\Gamma_{1}\right)}\right)=0
$$

Then

$$
\lim _{t \rightarrow \infty} \int_{U}\left(|\nabla p(x, t)|^{2-a}+|p(x, t)-\gamma(t)|^{2}\right) d x=0 .
$$

(ii) If in addition one has

$$
\lim _{t \rightarrow \infty}\left(\left|\gamma^{\prime \prime}(t)\right|+\left\|\phi_{t t}(\cdot, t)\right\|_{L^{2}\left(\Gamma_{1}\right)}\right)=0
$$

then

$$
\lim _{t \rightarrow \infty} \int_{U}\left(|\nabla p(x, t)|^{2-a}+|p(x, t)-\gamma(t)|^{2}+\left|p_{t}(x, t)\right|^{2}\right) d x=0 .
$$

In particular, let $p_{\gamma}(x, t)$ be the solution corresponding to the case $\psi(x, t)=\gamma(t)$. Then (4.13) and (4.15) hold for $p(x, t)=p_{\gamma}(x, t)$. Consequently, we have the asymptotic stability:

$$
\lim _{t \rightarrow \infty} \int_{U}\left|p(x, t)-p_{\gamma}(x, t)\right|^{2} d x=0,
$$

for any perturbed solution $p(x, t)$ as in Corollary 4.4 ,

Proof of Corollary 4.4. Set the extension $\Psi(x, t)$ to be $\gamma(t)+\Phi(x, t)$, where $\Phi(x, t)=$ $\mathcal{H}(\phi)$ - the harmonic extension of $\phi(x, t)$ defined in Remark 3.18. One can estimate $\Psi(x, t)$ as:

$$
\begin{aligned}
\|\Psi(\cdot, t)\|_{L^{2}(U)} & \leq C|\gamma(t)|+C\|\phi(\cdot, t)\|_{L^{2}\left(\Gamma_{1}\right)}, \\
\|\nabla \Psi(\cdot, t)\|_{L^{2}(U)} & \leq C\|\phi(\cdot, t)\|_{W^{1,2}\left(\Gamma_{1}\right)}, \\
\left\|\Psi_{t}(\cdot, t)\right\|_{L^{2}(U)} & \leq C\left|\gamma^{\prime}(t)\right|+C\left\|\phi_{t}(\cdot, t)\right\|_{L^{2}\left(\Gamma_{1}\right)}, \\
\left\|\nabla \Psi_{t}(\cdot, t)\right\|_{L^{2}(U)} & \leq C\left\|\phi_{t}(x, t)\right\|_{W^{1,2}\left(\Gamma_{1}\right)}, \\
\left\|\Psi_{t t}(\cdot, t)\right\|_{L^{2}(U)} & \leq C\left|\gamma^{\prime \prime}(t)\right|+C\left\|\phi_{t t}(\cdot, t)\right\|_{L^{2}\left(\Gamma_{1}\right)} .
\end{aligned}
$$

Note also that $\lim _{t \rightarrow \infty}\|\Phi(\cdot, t)\|_{L^{2}}=0$.

(i) From the estimates in (4.17) and (4.12), one easily verify (a)-(c) in Proposition 4.2. Therefore

$$
\lim _{t \rightarrow \infty} \int_{U}\left(|\nabla p(x, t)|^{2-a}+|p(x, t)-\Psi(x, t)|^{2}\right) d x=0 .
$$

Since

$$
\lim _{t \rightarrow \infty} \int_{U}|\Psi(x, t)-\gamma(t)|^{2} d x=\lim _{t \rightarrow \infty} \int_{U}|\Phi(x, t)|^{2} d x=0
$$

one obtains (4.13).

(ii) The proof of (4.15) is similar noting that (4.17) and (4.14) imply (d) in Proposition 4.3.. 
Example 4.5. In the previous corollary, let $\gamma(t)=A t^{\beta}$ with $\beta<1$, i.e.,

$$
\psi(x, t)=A t^{\beta}+\phi(x, t) \text { on } \Gamma_{1},
$$

then from (4.18) and (4.19) one has

$$
\lim _{t \rightarrow \infty} \int_{U}|\nabla p(x, t)|^{2-a}+\left|p(x, t)-A t^{\beta}\right|^{2} d x=0 .
$$

If, in addition, one has $\lim _{t \rightarrow \infty}\left\|\phi_{t t}(\cdot, t)\right\|_{L^{2}\left(\Gamma_{1}\right)}=0$ then

$$
\lim _{t \rightarrow \infty} \int_{U}|\nabla p(x, t)|^{2-a}+\left|p(x, t)-A t^{\beta}\right|^{2}+p_{t}^{2}(x, t) d x=0 .
$$

Remark 4.6. As one can see that the limit (4.4) makes sense only when any two extensions $\Psi_{1}$ and $\Psi_{2}$ satisfying (a)-(c) of the same boundary data $\psi$ converge to each other as $t \rightarrow \infty$. If that is the case then the limit in (4.4) does not depend on such an extension. This fact is indeed guaranteed by condition (a) and Poincare inequality:

$$
\begin{aligned}
\left\|\Psi_{1}(\cdot, t)-\Psi_{2}(\cdot, t)\right\|_{L^{2}} \leq C & \left\|\nabla \Psi_{1}(\cdot, t)-\nabla \Psi_{2}(\cdot, t)\right\|_{L^{2}} \\
& \leq C\left(\left\|\nabla \Psi_{1}(\cdot, t)\right\|_{L^{2}}+\left\|\nabla \Psi_{2}(\cdot, t)\right\|_{L^{2}}\right) \rightarrow 0 \text { as } t \rightarrow \infty
\end{aligned}
$$

However, there are cases so that $\|p(\cdot, t)-\Psi(\cdot, t)\|_{L^{2}} \rightarrow 0$ even when $\Psi(x, t)$ does not satisfy (a)-(c). For instance, in contrast to Example 4.5 above, let $\psi(x, t)=t$. Set $\Psi_{1}(x, t)=t+W(x)$ - the corresponding pseudo-steady state solution, where $W(x) \not \equiv 0$ is the basic pseudo-steady state profile (c.f. [1]). It is proved in [1] (see Theorem VII.3 and Example VII.5 with $\gamma(t)=t$ and $\varphi(x)=0$ ) that any solution $p(x, t)$ to the IBVP with this boundary data $\psi(x, t)$ satisfies $\lim _{t \rightarrow \infty}\left\|p(x, t)-\Psi_{1}(x, t)\right\|_{L^{2}}=0$. Note that $\Psi_{1}(x, t)$ satisfies neither condition (a) nor (b). Obviously, $\psi(x, t)$ also admits another extension $\Psi_{2}(x, t)=t$ which does not satisfy $\lim _{t \rightarrow \infty}\left\|\Psi_{2}(\cdot, t)-\Psi_{1}(\cdot, t)\right\|=0$.

4.2. Continuous dependence on the boundary data. We now study the structural stability of the IBVP $(3.1 \mathrm{a})-(3.1 \mathrm{~d})$ with respect to general boundary data $\psi(x, t)$. Let $p_{1}(x, t)$ and $p_{2}(x, t)$ be two solutions of the IBVP (3.1a)-(3.1d) with the boundary profiles $\psi_{1}(x, t)$ and $\psi_{2}(x, t)$, respectively.

Let $\Psi_{k}(x, t)$ be an extension of $\psi_{k}(x, t)$, for $k=1,2$.

We denote

$$
\begin{gathered}
z(x, t)=p_{1}(x, t)-p_{2}(x, t), \quad \Psi(x, t)=\Psi_{1}(x, t)-\Psi_{2}(x, t), \\
\bar{p}_{k}=p_{k}-\Psi_{k}, \quad k=1,2, \quad \bar{z}=\bar{p}_{1}-\bar{p}_{2}=z-\Psi .
\end{gathered}
$$

Let $H_{k}(x, t)=H\left[p_{k}\right](x, t)=H\left(\left|\nabla p_{k}(x, t)\right|\right)$ for $k=1,2$.

Recall that $a=\frac{\operatorname{deg}(g)}{\operatorname{deg}(g)+1}$. Let $b=\frac{a}{2-a}=\frac{\operatorname{deg}(g)}{\operatorname{deg}(g)+2}$.

We will establish various estimates for $\bar{Z}(t) \stackrel{\text { def }}{=} \int_{U} \bar{z}^{2}(x, t) d x$, for $t \geq 0$. First, we derive a general differential inequality for $\bar{z}$. 
Lemma 4.7. One has for all $t \geq 0$,

$$
\begin{aligned}
\frac{1}{2} \frac{d}{d t} \int_{U} \bar{z}^{2} d x \leq & -C\left(\int_{U}|\nabla \bar{z}|^{2-a} d x\right)^{\frac{2}{2-a}}\left(1+\left\|H_{1}\right\|_{L^{1}}+\left\|H_{2}\right\|_{L^{1}}\right)^{-b} \\
& +C\left(1+\left\|H_{1}\right\|_{L^{1}}+\left\|H_{2}\right\|_{L^{1}}\right)^{-b}\|\nabla \Psi\|_{L^{2-a}}^{2} \\
& +C\left(\left\|H_{1}\right\|_{L^{1}}+\left\|H_{2}\right\|_{L^{1}}\right)^{1 / 2}\|\nabla \Psi\|_{L^{2}} \\
& +C\left(1+\left\|H_{1}\right\|_{L^{1}}+\left\|H_{2}\right\|_{L^{1}}\right)^{b}\left\|\Psi_{t}\right\|_{L^{r_{0}}}^{2}
\end{aligned}
$$

where $r_{0}$ is defined by (3.6) and the constants depend on $\chi(\vec{a})$.

Proof. Note that $\left.\bar{z}\right|_{\Gamma_{1}}=0$. Using Eq. (3.2) for each $\bar{p}_{k}$, one easily finds

$$
\begin{aligned}
& \frac{1}{2} \frac{d}{d t} \int_{U} \bar{z}^{2} d x=-\int_{U}\left(K\left(\left|\nabla p_{1}\right|\right) \nabla p_{1}-K\left(\left|\nabla p_{2}\right|\right) \nabla p_{2}\right) \cdot\left(\nabla p_{1}-\nabla p_{2}\right) d x \\
& \quad+\int_{U} K\left(\left|\nabla p_{1}\right|\right) \nabla p_{1} \cdot \nabla \Psi d x-\int_{U} K\left(\left|\nabla p_{2}\right|\right) \nabla p_{2} \cdot \nabla \Psi d x-\int_{U} \Psi_{t} \bar{z} d x .
\end{aligned}
$$

By (2.27) and Holder inequality, one derives

$$
\begin{aligned}
& \frac{1}{2} \frac{d}{d t} \int_{U} \bar{z}^{2} d x \\
& \leq-C\left[\int_{U}\left|\nabla\left(p_{1}-p_{2}\right)\right|^{2-a} d x\right]^{\frac{2}{2-a}}\left[1+\max \left(\left\|\nabla p_{1}\right\|_{L^{2-a}},\left\|\nabla p_{2}\right\|_{L^{2-a}}\right)\right]^{-a} \\
& \quad+C_{2}\left(\left\|H_{1}\right\|_{L^{1}}^{1 / 2}+\left\|H_{2}\right\|_{L^{1}}^{1 / 2}\right)\|\nabla \Psi\|_{L^{2}}+\int_{U}\left|\Psi_{t} \| \bar{z}\right| d x .
\end{aligned}
$$

Hence by virtue of the relation $(2.25)$ applied for $\left|\nabla p_{k}\right|^{2-a}$ and $H_{k}$

$$
\begin{aligned}
\frac{1}{2} \frac{d}{d t} \int_{U} \bar{z}^{2} d x \leq & -C\left[\int_{U}\left|\nabla\left(p_{1}-p_{2}\right)\right|^{2-a} d x\right]^{\frac{2}{2-a}}\left(1+\left\|H_{1}\right\|_{L^{1}}+\left\|H_{2}\right\|_{L^{1}}\right)^{-b} \\
& +C_{2}\left(\left\|H_{1}\right\|_{L^{1}}^{1 / 2}+\left\|H_{2}\right\|_{L^{1}}^{1 / 2}\right)\|\nabla \Psi\|_{L^{2}}+\int_{U}\left|\Psi_{t} \| \bar{z}\right| d x \\
\leq & -C\left[\int_{U}|\nabla \bar{z}|^{2-a} d x\right]^{\frac{2}{2-a}}\left(1+\left\|H_{1}\right\|_{L^{1}}+\left\|H_{2}\right\|_{L^{1}}\right)^{-b} \\
& +C\|\nabla \Psi\|_{L^{2-a}}^{2}\left(1+\left\|H_{1}\right\|_{L^{1}}+\left\|H_{2}\right\|_{L^{1}}\right)^{-b} \\
& +C_{2}\left(\left\|H_{1}\right\|_{L^{1}}^{1 / 2}+\left\|H_{2}\right\|_{L^{1}}^{1 / 2}\right)\|\nabla \Psi\|_{L^{2}}+\int_{U}\left|\Psi_{t} \| \bar{z}\right| d x
\end{aligned}
$$

By Holder, Sobolev and then Cauchy inequalities one obtains

$$
\begin{aligned}
\int_{U}\left|\Psi_{t}\right||\bar{z}| d x \leq & \left\|\Psi_{t}\right\|_{L^{r}}\|\bar{z}\|_{L^{(2-a)^{*}}} \leq C\left\|\Psi_{t}\right\|_{L^{r}}\|\nabla \bar{z}\|_{L^{2-a}} \\
\leq & C \varepsilon\left(1+\left\|H_{1}\right\|_{L^{1}}+\left\|H_{2}\right\|_{L^{1}}\right)^{-b}\|\nabla \bar{z}\|_{L^{2-a}}^{2} \\
& +C_{\varepsilon}\left(1+\left\|H_{1}\right\|_{L^{1}}+\left\|H_{2}\right\|_{L^{1}}\right)^{b}\left\|\Psi_{t}\right\|_{L^{r_{0}}}^{2}
\end{aligned}
$$

Using this estimate for the RHS of (4.27), and choosing an appropriate $\varepsilon$ one obtains (4.25). 
It follows from Lemma 4.7 that the solutions of IBVP (3.1a)-(3.1d) continuously depend on the initial and boundary data, (in any finite time intervals), without any restrictions on the degree of the Forchheimer polynomial.

Let $G_{j}\left[\Psi_{k}\right], k=1,2, j=1,2,3,4$, denote the quantity $G_{j}$, defined in (3.5), (3.18), (3.20), and (3.46) for corresponding solution $p_{k}$ with boundary data extension $\Psi_{k}$.

Similarly, let $\Lambda_{j}\left[\Psi_{k}\right], k=1,2, j=1,2$, denote the corresponding quantity $\Lambda_{j}$ defined in (3.10) and (3.12) for $\Psi_{k}$.

Let $\bar{m}(t)=\bar{m}_{1}(t)+\bar{m}_{2}(t)$, where for $k=1,2$,

$$
\begin{aligned}
\bar{m}_{k}(t)= & e^{-C_{1} t} \int_{U} H_{k}(x, 0) d x+\int_{U} \bar{p}_{k}^{2}(x, 0) d x \\
& +\int_{0}^{t} e^{-C_{1}(t-\tau)}\left(\Lambda_{1}\left[\Psi_{k}\right](\tau)+G_{3}\left[\Psi_{k}\right](\tau)\right) d \tau,
\end{aligned}
$$

with $C_{1}$ being the positive constant in Corollary 3.7.

Theorem 4.8. One has for all $t \geq 0$ that

$$
\begin{aligned}
& \int_{U} \bar{z}^{2}(x, t) d x \leq \int_{U} \bar{z}^{2}(x, 0) d x+C \int_{0}^{t}\left(\|\nabla \Psi(\cdot, \tau)\|_{L^{2-a}}^{2}\right. \\
& \left.+\bar{m}(\tau)^{1 / 2}\|\nabla \Psi(\cdot, \tau)\|_{L^{2}}+(1+\bar{m}(\tau))^{b}\left\|\Psi_{t}(\cdot, \tau)\right\|_{L^{r_{0}}}^{2}\right) d \tau
\end{aligned}
$$

Consequently, for any give $T>0$,

$$
\begin{array}{r}
\sup _{[0, T]} \int_{U} z^{2}(x, t) d x \leq 4 \int_{U} z^{2}(x, 0) d x+6 \sup _{[0, T]}\|\Psi(\cdot, t)\|_{L^{2}}^{2}+C T \sup _{[0, T]}\|\nabla \Psi(\cdot, t)\|_{L^{2-a}}^{2} \\
+C T\left(1+A_{*}+D_{*}(T)\right)^{\delta}\left(\sup _{[0, T]}\|\nabla \Psi(\cdot, t)\|_{L^{2}}+\sup _{[0, T]}\left\|\Psi_{t}(\cdot, t)\right\|_{L^{r_{0}}}^{2}\right),
\end{array}
$$

where $\delta=\max \{1 / 2, b\}$,

$$
\begin{gathered}
A_{*}=\int_{U} H_{1}(x, 0) d x+\int_{U} \bar{p}_{1}^{2}(x, 0) d x+\int_{U} H_{2}(x, 0) d x+\int_{U} \bar{p}_{2}^{2}(x, 0) d x, \\
D_{*}(T)=\sum_{k=1}^{2} \sup _{[0, T]} \int_{0}^{t} e^{-C_{1}(t-\tau)}\left(\Lambda_{1}\left[\Psi_{k}\right](\tau)+G_{3}\left[\Psi_{k}\right](\tau)\right) d \tau .
\end{gathered}
$$

Proof. By virtue of estimate (3.29) in Corollary 3.7, one has $\left\|H_{k}(\cdot, t)\right\|_{L^{1}} \leq C \bar{m}_{k}(t)$. Neglecting the negative term on the RHS of (4.25) one obtains

$$
\frac{d}{d t} \int_{U} \bar{z}^{2}(x, t) d x \leq C_{1}\|\nabla \Psi\|_{L^{2-a}}^{2}+C_{2} \bar{m}(t)^{1 / 2}\|\nabla \Psi\|_{L^{2}}+C_{3}(1+\bar{m}(t))^{b}\left\|\Psi_{t}\right\|_{L^{r}}^{2} .
$$

Integrating this differential inequality from 0 to $t$ yields (4.29).

Let $T>0$. Note for $t \in[0, T]$ that $\bar{m}(t) \leq A_{*}+D_{*}(T)$. From (4.29) it follows

$$
\begin{aligned}
& \sup _{[0, T]} \int_{U} \bar{z}^{2}(x, t) d x \leq \int_{U} \bar{z}^{2}(x, 0) d x+C T \sup _{[0, T]}\|\nabla \Psi(\cdot, t)\|_{L^{2-a}}^{2} \\
& +C T\left(1+A_{*}+D_{*}(T)\right)^{\delta}\left(\sup _{[0, T]}\|\nabla \Psi(\cdot, t)\|_{L^{2}}+\sup _{[0, T]}\left\|\Psi_{t}(\cdot, t)\right\|_{L^{r_{0}}}^{2}\right) .
\end{aligned}
$$

One then obtains (4.30) by applying Cauchy inequalities: $z^{2} \leq 2\left(\bar{z}^{2}+\Psi^{2}\right)$ and $\bar{z}^{2} \leq 2\left(z^{2}+\Psi^{2}\right)$. 
In particular, when growth rates of different norms of $\Psi_{k}$ are specified, one has:

Corollary 4.9. Suppose for both $k=1,2$ and $t \geq 0$ one has

$$
\left\|\nabla \Psi_{k}(\cdot, t)\right\|_{L^{2}}^{2}+\left\|\left(\Psi_{k}\right)_{t}(\cdot, t)\right\|_{L^{r_{0}}}^{\frac{2-a}{1-a}} \leq C(1+t)^{r_{1}}
$$

and

$$
\left\|\nabla\left(\Psi_{k}\right)_{t}(\cdot, t)\right\|_{L^{2}}^{2}+\left\|\left(\Psi_{k}\right)_{t}(\cdot, t)\right\|_{L^{2}}^{2} \leq C(1+t)^{r_{2}},
$$

where $r_{1}, r_{2}>0$. Let $r_{3}=1+\max \left\{r_{1}+1, r_{2}\right\}$. Then

$$
\begin{aligned}
\int_{U} \bar{z}^{2}(x, t) d x & \leq \int_{U} \bar{z}^{2}(x, 0) d x \\
& +C_{*} \int_{0}^{t}(1+\tau)^{r_{3} / 2}\|\nabla \Psi(\cdot, \tau)\|_{L^{2}}+(1+\tau)^{r_{3} b}\left\|\Psi_{t}(\cdot, \tau)\right\|_{L^{r}}^{2} d \tau
\end{aligned}
$$

where $C_{*}$ depends also on the initial data of the solutions $p_{1}$ and $p_{2}$.

Proof. One easily finds

$$
G_{1}\left[p_{k}\right](t) \leq C\left(1+\int_{U}|\nabla \Psi|^{2} d x+\left(\int_{U}\left|\Psi_{t}\right|^{r_{0}} d x\right)^{\frac{2-a}{r_{0}(1-a)}}\right) \leq C(1+t)^{r_{1}} .
$$

Similarly,

$$
\begin{aligned}
& G_{2}\left[\Psi_{k}\right](t) \leq C(1+t)^{r_{2}}, \\
& \Lambda_{1}\left[\Psi_{k}\right](t) \leq C(1+t)^{r_{1}+1} \leq C(1+t)^{r_{3}-1}, \\
& G_{3}\left[\Psi_{k}\right](t) \leq C(1+t)^{r_{3}-1} .
\end{aligned}
$$

Therefore

$$
\bar{m}(t) \leq C_{*}+C(1+t)^{r_{3}} \leq C_{*}(1+t)^{r_{3}} .
$$

Note also that

$$
\|\nabla \Psi\|_{L^{2-a}}^{2} \leq C\left(\left\|\nabla \Psi_{1}\right\|_{L^{2}}+\left\|\nabla \Psi_{2}\right\|_{L^{2}}\right)\|\nabla \Psi\|_{L^{2}} \leq C(1+t)^{r_{3} / 2}\|\nabla \Psi\|_{L^{2}} .
$$

Then (4.37) follows the estimate (4.29).

For the asymptotic stability of the solutions with respect to the boundary data, we will use Lemma 4.7 and estimate (3.30) for functions $H\left[p_{k}\right],(k=1,2)$. Therefore we denote

$$
\begin{aligned}
m_{k}(t)= & e^{-C_{1} t} \int_{U} H_{k}(x, 0) d x+\int_{U} \bar{p}_{k}^{2}(x, 0) d x \\
& +\int_{0}^{t} e^{-C_{1}(t-\tau)}\left(\Lambda_{2}\left[\Psi_{k}\right](\tau)+G_{3}\left[\Psi_{k}\right](\tau)\right) d \tau, \quad k=1,2 .
\end{aligned}
$$

Again $C_{1}$ here is the same constant $C_{1}$ in Corollary 3.7. Also, let

$$
m(t)=m_{1}(t)+m_{2}(t) \text { and } S\left(t^{\prime}, t\right)=\int_{t^{\prime}}^{t}(1+m(\tau))^{-b} d \tau .
$$

One then has: 
Theorem 4.10. Suppose $\operatorname{deg}(g) \leq 4 /(n-2)$. Then for all $t \geq 0$ one has

$$
\begin{aligned}
\int_{U} \bar{z}^{2}(x, t) d x \leq & e^{-C_{1} S(0, t)} \int_{U} \bar{z}^{2}(x, 0) d x \\
& +C \int_{0}^{t} e^{-C_{1} S(\tau, t)}\left(\|\nabla \Psi(\cdot, \tau)\|_{L^{2-a}}^{2}\right. \\
& \left.+m(\tau)^{1 / 2}\|\nabla \Psi(\cdot, \tau)\|_{L^{2}}+(1+m(\tau))^{b}\left\|\Psi_{t}(\cdot, \tau)\right\|_{L^{r_{0}}}^{2}\right) d \tau .
\end{aligned}
$$

Proof. From (4.25) one easily finds

$$
\begin{aligned}
\frac{1}{2} \frac{d}{d t} \int_{U} \bar{z}^{2} d x \leq & -C\left(\int_{U}|\nabla \bar{z}|^{2-a} d x\right)^{\frac{2}{2-a}}\left(1+\left\|H_{1}\right\|_{L^{1}}+\left\|H_{2}\right\|_{L^{1}}\right)^{-b} \\
& +C\left(1+\left\|H_{1}\right\|_{L^{1}}+\left\|H_{2}\right\|_{L^{1}}\right)^{-b}\|\nabla \Psi\|_{L^{2-a}}^{2} \\
& +C\left(\left\|H_{1}\right\|_{L^{1}}+\left\|H_{2}\right\|_{L^{1}}\right)^{1 / 2}\|\nabla \Psi\|_{L^{2}} \\
& +C\left(1+\left\|H_{1}\right\|_{L^{1}}+\left\|H_{2}\right\|_{L^{1}}\right)^{b}\left\|\Psi_{t}\right\|_{L^{r_{0}}}^{2}
\end{aligned}
$$

The condition on $\operatorname{deg}(g)$ implies $2 \leq(2-a)^{*}$, hence one has the Sobolev inequality:

$$
\|\bar{z}\|_{L^{2}} \leq C\|\nabla \bar{z}\|_{L^{2-a}}
$$

Using (4.42) in RHS of the equation (4.41) one obtains

$$
\begin{aligned}
\frac{d}{d t} \bar{Z}(t) \leq & -C \bar{Z}(t)(1+m(t))^{-b}+C\|\nabla \Psi\|_{L^{2-a}}^{2} \\
& +C m(t)^{1 / 2}\|\nabla \Psi\|_{L^{2}}+(1+m(t))^{b}\left\|\Psi_{t}\right\|_{L^{r_{0}}}^{2} .
\end{aligned}
$$

Applying Gronwall inequality to (4.43) yields (4.40).

The Lyapunov stability immediately follows for a class of the individual $\Psi_{k}(x, t)$. In particular one has:

Corollary 4.11. Suppose $\operatorname{deg}(g) \leq 4 /(n-2)$. Assume that

$$
\sum_{k=1}^{2}\left(\sup _{[0, \infty)} \Lambda_{2}\left[\Psi_{k}\right](t)+\sup _{[0, \infty)} G_{3}\left[\Psi_{k}\right](t)\right)<\infty
$$

Then one has

$$
\begin{aligned}
& \sup _{[0, \infty)} \int_{U} z^{2}(x, t) d x \leq 4 \int_{U} z^{2}(x, 0) d x+C \sup _{[0, \infty)}\|\Psi(\cdot, t)\|_{L^{2}}^{2} \\
& \quad+C \sup _{[0, \infty)}\left(A_{*}^{b}\|\nabla \Psi(\cdot, t)\|_{L^{2-a}}^{2}+A_{*}^{b+\frac{1}{2}}\|\nabla \Psi(\cdot, t)\|_{L^{2}}+A_{*}^{2 b}\left\|\Psi_{t}(\cdot, t)\right\|_{L^{r_{0}}}^{2}\right)
\end{aligned}
$$

where

$$
A_{*}=1+\sum_{k=1}^{2}\left(\int_{U}\left|\nabla p_{k}(x, 0)\right|^{2-a}+\bar{p}_{k}^{2}(x, 0) d x+\sup _{[0, \infty)} \Lambda_{2}\left[\Psi_{k}\right](t)+\sup _{[0, \infty)} G_{3}\left[\Psi_{k}\right](t)\right)<\infty .
$$


Proof. One has $m(t) \leq C A_{*}$ and hence $S(\tau, t) \geq C A_{*}^{-b}(t-\tau)$. Therefore, by the virtue of (4.40), it follows that

$$
\begin{aligned}
\sup _{[0, \infty)} \int_{U} \bar{z}^{2}(x, t) d x \leq \int_{U} \bar{z}^{2}(x, 0) d x+ & C \sup _{[0, \infty)}\left(A_{*}^{b}\|\nabla \Psi(\cdot, t)\|_{L^{2-a}}^{2}\right. \\
& \left.+A_{*}^{b+\frac{1}{2}}\|\nabla \Psi(\cdot, t)\|_{L^{2}}+A_{*}^{2 b}\left\|\Psi_{t}(\cdot, t)\right\|_{L^{r_{0}}}^{2}\right) .
\end{aligned}
$$

Then (4.45) follows by Cauchy inequality.

Furthermore, for the asymptotic stability, one has:

Corollary 4.12. Suppose $\operatorname{deg}(g) \leq 4 /(n-2)$. Assume that

$$
\begin{gathered}
\qquad \lim _{t \rightarrow \infty} S(0, t)=\infty, \\
\lim _{t \rightarrow \infty}(1+m(t))^{b+\frac{1}{2}}\|\nabla \Psi(\cdot, t)\|_{L^{2}}=0, \quad \lim _{t \rightarrow \infty}(1+m(t))^{b}\left\|\Psi_{t}(\cdot, t)\right\|_{L^{r_{0}}}=0 .
\end{gathered}
$$

Then $\lim _{t \rightarrow \infty} \int_{U} \bar{z}^{2}(x, t) d x=0$.

Proof. The first term on the RHS of (4.40), under condition (4.46), obviously converges to zero. Next let us rewrite the second term on the RHS of (4.40) as $e^{-C_{1} S(0, t)} J(t)$ where

$$
\begin{aligned}
J(t)= & \int_{0}^{t} e^{C_{1} S(0, \tau)}\left[\|\nabla \Psi(\cdot, \tau)\|_{L^{2-a}}^{2}+m(\tau)^{1 / 2}\|\nabla \Psi(\cdot, \tau)\|_{L^{2}}\right. \\
& \left.+(1+m(\tau))^{b}\left\|\Psi_{t}(\cdot, \tau)\right\|_{L^{r}}^{2}\right] d \tau .
\end{aligned}
$$

In case $J(t)$ is bounded, one has $\bar{Z}(t)=\int_{U} \bar{z}^{2}(x, t) d x \rightarrow 0$ thanks to $e^{-C_{1} S(0, t)} \rightarrow$ 0 as $t \rightarrow \infty$.

In case $\lim _{t \rightarrow \infty} J(t)=\infty$, applying the Hôpital rule and condition (4.47), to the term $e^{-C_{1} S(0, t)} J(t)$ noting that

$$
(1+m(t))^{b}\|\nabla \Psi(\cdot, t)\|_{L^{2-a}}^{2} \leq(1+m(t))^{2\left(b+\frac{1}{2}\right)}\|\nabla \Psi(\cdot, t)\|_{L^{2}}^{2},
$$

one again asserts $\bar{Z}(t) \rightarrow 0$ as $t \rightarrow \infty$.

An interesting case from application point of view is the following generalization of the pseudo-steady state boundary conditions $[2,3,1]$.

Corollary 4.13. Suppose $\operatorname{deg}(g) \leq 4 /(n-2)$ and the boundary data $\psi_{k}(x, t)=$ $\gamma_{k}(t)+\phi_{k}(x, t)$ on $\Gamma_{1}$. Assume

$$
\left|\gamma_{k}^{\prime}(t)\right|=O\left(t^{r}\right) \text { as } t \rightarrow \infty,
$$

$$
\left\|\phi_{k}(\cdot, t)\right\|_{W^{1,2}\left(\Gamma_{1}\right)},\left\|\left(\phi_{k}\right)_{t}(\cdot, t)\right\|_{W^{1,2}\left(\Gamma_{1}\right)} \leq C, \quad \text { for all } t \geq 0,
$$

for $k=1,2$. Let $\phi=\phi_{1}-\phi_{2}$ and $\gamma=\gamma_{1}-\gamma_{2}$. Then

$$
\lim _{t \rightarrow \infty} \int_{U}|z(x, t)-\gamma(t)|^{2} d x=0
$$

if either

(i) the exponent $r<0$ and

$$
\lim _{t \rightarrow \infty}\|\phi(\cdot, t)\|_{W^{1,2}\left(\Gamma_{1}\right)}=\lim _{t \rightarrow \infty}\left\|\phi_{t}(\cdot, t)\right\|_{W^{1,2}\left(\Gamma_{1}\right)}=0 ;
$$

or, 
(ii) the exponent $r \geq 0$ satisfies

$$
r<\frac{2+a^{2}}{a}-\frac{3}{2}
$$

and

$$
\lim _{t \rightarrow \infty} t^{\frac{r(2 b+1)}{1-a}}\|\phi(\cdot, t)\|_{W^{1,2}\left(\Gamma_{1}\right)}=\lim _{t \rightarrow \infty} t^{\frac{2 r b}{1-a}}\left\|\phi_{t}(\cdot, t)\right\|_{W^{1,2}\left(\Gamma_{1}\right)}=\lim _{t \rightarrow \infty} t^{\frac{2 r b}{1-a}} \gamma^{\prime}(t)=0 .
$$

Proof. Let $\Phi_{k}(x, t)$ be the harmonic extension $\mathcal{H}\left(\phi_{k}\right)$ defined in Remark 3.18. Note that

$$
\left\|\Phi_{k}(\cdot, t)\right\|_{L^{2}(U)}+\left\|\left(\Phi_{k}\right)_{t}(\cdot, t)\right\|_{L^{2}(U)} \leq C .
$$

Let the extension $\Psi_{k}$ of $\psi_{k}$ be $\gamma_{k}+\Phi_{k}$. Let $\Phi=\Phi_{1}-\Phi_{2}$ and $\Psi=\Psi_{1}-\Psi_{2}=\gamma+\Phi$. One has $\bar{z}=z-\gamma-\Phi$.

(i) Case $r<0$ : One has

$$
G_{1}\left[\Psi_{k}\right] \leq C, G_{2}\left[\Psi_{k}\right] \leq C, \Lambda_{2}\left[\Psi_{k}\right] \leq C .
$$

Consequently, $m(t) \leq C$ hence (4.46) holds. Also, (4.51) implies (4.47). By Corollary 4.12 , one then has $\int_{U} \bar{z}^{2}(x, t) d x \rightarrow 0$ as $t \rightarrow \infty$. Since $\|\Phi(\cdot, t)\|_{L^{2}} \rightarrow 0$ as $t \rightarrow \infty$, this implies (4.50).

(ii) Case $r \geq 0$ : Using the estimates in Remark 3.18, one finds

$$
\begin{gathered}
G_{1}\left[\Psi_{k}\right] \leq C(1+t)^{r(2-a) /(1-a)}, G_{2} \leq C(1+t)^{2 r}, \\
\Lambda_{2}\left[\Psi_{k}\right] \leq C(1+t)^{2 r /(1-a)} .
\end{gathered}
$$

Hence $m(t) \leq C(1+t)^{\frac{2 r}{1-a}}$. For $t \geq T_{0} \gg 1$ one has

$$
S(0, t) \geq C \int_{T_{0}}^{t} \tau^{-b \frac{2 r}{1-a}} d \tau \geq C t^{-\frac{2 r b}{1-a}+1} .
$$

Condition (4.52) is equivalent to $r<\frac{(1-a)(2-a)}{2 a}=\frac{1-a}{2 b}$, hence $1-\frac{2 r b}{1-a}>0$ and $\lim _{t \rightarrow \infty} S(0, t)=\infty$. Since $\Phi=\mathcal{H}(\phi)$, by using the estimate (4.17) for $\Phi$ and condition (4.53) one obtains (4.47). Applying Corollary 4.12 once again gives the convergence $\lim _{t \rightarrow \infty} \int_{U} \bar{z}^{2}(x, t) d x=0$. The limit (4.50) then follows by this and the fact that $\lim _{t \rightarrow \infty}\|\Phi(\cdot, t)\|_{L^{2}}=0$.

\section{Dependence on the Forchheimer Polynomials}

In this section, we study the continuous dependence of the solutions to IBVP (3.1a)-(3.1d) on the coefficient vector $\vec{a}$ of the Forchheimer polynomials $g(s, \vec{a})$.

Let $N$ and the exponent vector $\vec{\alpha}$ be fixed. Let the Forchheimer polynomial $g(s, \vec{a})$ belong to the class $\operatorname{FP}(N, \vec{\alpha})$. First we calculate partial derivatives of the function $K(\xi, \vec{a})$ defined by (2.13) with respect to all variables.

Lemma 5.1. One has for $\xi \geq 0$ and $\vec{a}=\left(a_{0}, a_{1}, \ldots, a_{N}\right)$ that

$$
\begin{aligned}
& K_{\xi}(\xi, \vec{a})=-K(\xi, \vec{a}) \frac{g_{s}}{g^{2}(s, \vec{a})+\xi g_{s}(s, \vec{a})}, \\
& K_{a_{i}}(\xi, \vec{a})=-K(\xi, \vec{a}) \frac{g_{a_{i}}(s, \vec{a})}{g(s, \vec{a})+s g_{s}(s, \vec{a})},
\end{aligned}
$$

where $s=s(\xi, \vec{a})$ is defined by (2.12). 
Proof. Note that

$$
g_{s}(s, \vec{a})=\sum_{i=0}^{N} a_{i} \alpha_{i} s^{\alpha_{i}-1} \quad \text { and } \quad g_{a_{i}}(s, \vec{a})=s^{\alpha_{i}} .
$$

Taking derivative of (2.12) with respect to $\xi$ one has

$$
s_{\xi} g(s, \vec{a})+s g_{s}(s, \vec{a}) s_{\xi}=1,
$$

hence

$$
\frac{\partial s}{\partial \xi}(\xi, \vec{a})=\left.\frac{1}{g(s, \vec{a})+s g_{s}(s, \vec{a})}\right|_{s=s(\xi, \vec{a})} .
$$

Therefore the partial derivative of $K(\xi, \vec{a})$ with respect to $\xi$ is

$$
K_{\xi}(\xi, \vec{a})=-\frac{g_{s}(s, \vec{a}) s_{\xi}(\xi, \vec{a})}{g^{2}(s, \vec{a})}=-K(\xi, \vec{a}) \frac{g_{s}(\xi, \vec{a})}{g^{2}+\xi g_{s}} .
$$

Thus one obtains (5.1). Similarly, taking derivative of (2.12) with respect to $a_{i}$ one has

$$
s_{a_{i}} g(s, \vec{a})+s g_{s}(s, \vec{a}) s_{a_{i}}+s g_{a_{i}}(s, \vec{a})=0
$$

hence

$$
\begin{gathered}
\frac{\partial s}{\partial a_{i}}(\xi, \vec{a})=\left.\frac{-s g_{a_{i}}}{g(s, \vec{a})+s g_{s}(s, \vec{a})}\right|_{s=s(\xi, \vec{a})} \\
K_{a_{i}}(\xi, \vec{a})=-\frac{g_{s} s_{a_{i}}+g_{a_{i}}}{g^{2}}=-K(\xi, \vec{a}) \frac{g_{s}\left(\frac{-s g_{a_{i}}}{g+s g_{s}}\right)+g_{a_{i}}}{g}=-K(\xi, \vec{a}) \frac{g_{a_{i}} g}{g\left(g+s g_{s}\right)} .
\end{gathered}
$$

Therefore one obtains (5.2).

Let $\vec{a}$ and $\vec{a}^{\prime}$ be two arbitrary vectors. We denote by $\vec{a} \vee \vec{a}^{\prime}$ and $\vec{a} \wedge \vec{a}^{\prime}$ the maximum and minimum vectors of the two, respectively, with components

$$
\left(\vec{a} \vee \vec{a}^{\prime}\right)_{j}=\max \left\{a_{j}, a_{j}^{\prime}\right\} \quad \text { and } \quad\left(\vec{a} \wedge \vec{a}^{\prime}\right)_{j}=\min \left\{a_{j}, a_{j}^{\prime}\right\}
$$

Then component-wise one has $\vec{a} \wedge \vec{a}^{\prime} \leq \vec{a}, \vec{a}^{\prime} \leq \vec{a} \vee \vec{a}^{\prime}$.

Define $\chi\left(\vec{a}, \vec{a}^{\prime}\right)=\max \left\{\chi(\vec{a}), \chi\left(\vec{a}^{\prime}\right)\right\}$. Note that

$$
\begin{gathered}
\chi\left(\vec{a} \vee \vec{a}^{\prime}\right), \quad \chi\left(\vec{a} \wedge \vec{a}^{\prime}\right) \leq \chi\left(\vec{a}, \vec{a}^{\prime}\right), \\
\chi\left(t \vec{a}+(1-t) \vec{a}^{\prime}\right) \leq \chi\left(\vec{a}, \vec{a}^{\prime}\right) \quad \forall t \in[0,1] .
\end{gathered}
$$

Perturbing the coefficient vector $\vec{a}$ in the monotonicity (2.26), one has the following version:

Lemma 5.2. Let $g(s, \vec{a})$ and $g\left(s, \vec{a}^{\prime}\right)$ belong to class $F P(N, \alpha)$. Then for any $y, y^{\prime}$ in $\mathbb{R}^{n}$, one has

$$
\begin{array}{r}
\left(K(|y|, \vec{a}) y-K\left(\left|y^{\prime}\right|, \vec{a}^{\prime}\right) y^{\prime}\right) \cdot\left(y-y^{\prime}\right) \geq(1-a) K\left(|y| \vee\left|y^{\prime}\right|, a \vee \vec{a}^{\prime}\right)\left|y-y^{\prime}\right|^{2} \\
-C_{0} \chi\left(\vec{a}, \vec{a}^{\prime}\right)\left|a-\vec{a}^{\prime}\right| K\left(|y| \vee\left|y^{\prime}\right|, a \wedge \vec{a}^{\prime}\right)\left(|y| \vee\left|y^{\prime}\right|\right)\left|y-y^{\prime}\right|
\end{array}
$$

where $a \in[0,1)$ is defined in (2.10), the positive constant $C_{0}$ depends on $N, \alpha_{N}$. 
Proof. Let $\gamma(t)=t y+(1-t) y^{\prime}$ and $\vec{b}(t)=\left(b_{0}, b_{1}, \ldots, b_{N}\right)(t)=t \vec{a}+(1-t) \vec{a}^{\prime}$ for $t \in[0,1]$. Note that $|y| \wedge\left|y^{\prime}\right| \leq|\gamma(t)| \leq|y| \vee\left|y^{\prime}\right|, a \wedge \vec{a}^{\prime} \leq \vec{b}(t) \leq \vec{a} \vee \vec{a}^{\prime}$ (component-wise) and $\chi(\vec{b}(t)) \leq \chi\left(\vec{a}, \vec{a}^{\prime}\right)$.

Let $F(t)=K(|\gamma(t)|, \vec{b}(t)) \gamma(t) \cdot\left(y-y^{\prime}\right)$. Then there is $t_{0}$ in $(0,1)$ such that

$$
\left(K(|y|, \vec{a}) y-K\left(\left|y^{\prime}\right|, \vec{a}^{\prime}\right) y^{\prime}\right) \cdot\left(y-y^{\prime}\right)=F(1)-F(0)=F^{\prime}\left(t_{0}\right) .
$$

In the calculations right below, $s=s(t)=s(|\gamma(t)|, \vec{b}(t))$ and $g=g(s(t), \vec{b}(t))$. Using the formulas in 5.1 one has

$$
\begin{aligned}
F^{\prime}(t)= & K(|\gamma(t)|, \vec{b}(t))\left|y-y^{\prime}\right|^{2}+K_{\xi}(|\gamma(t)|, \vec{b}(t)) \frac{\gamma \cdot \gamma^{\prime}}{|\gamma(t)|} \gamma(t) \cdot\left(y-y^{\prime}\right) \\
& +K_{a_{j}}(|\gamma(t)|, \vec{b}(t)) b_{j}^{\prime}(t) \gamma(t) \cdot\left(y-y^{\prime}\right) \\
= & K(|\gamma(t)|, \vec{b}(t))\left\{\left|y-y^{\prime}\right|^{2}-\frac{g_{s}}{g^{2}+|\gamma(t)| g_{s}} \cdot \frac{\left|\gamma(t) \cdot\left(y-y^{\prime}\right)\right|^{2}}{|\gamma(t)|}\right. \\
& \left.+\frac{\sum g_{a_{j}}\left(a_{j}-a_{j}^{\prime}\right)}{g+s g_{s}} \gamma(t) \cdot\left(y-y^{\prime}\right)\right\}
\end{aligned}
$$

Thus

$$
\begin{aligned}
F^{\prime}(t) \geq & K(|\gamma(t)|, \vec{b}(t))\left\{\left|y-y^{\prime}\right|^{2}-\frac{g_{s}}{g^{2}+|\gamma(t)| g_{s}}|\gamma(t)|\left|y-y^{\prime}\right|^{2}\right. \\
& \left.-\frac{\left|\sum g_{a_{j}}\left(a_{j}-a_{j}^{\prime}\right)\right|}{g+s g_{s}}|\gamma(t)|\left|y-y^{\prime}\right|\right\} .
\end{aligned}
$$

First, consider the case $N>0$. One has $\alpha_{N}>0$ and $g \geq \alpha_{N}^{-1} s g_{s}$. Hence

$$
g^{2}+|\gamma| g_{s} \leq g\left(\alpha_{N}^{-1} s g_{s}\right)+|\gamma| g_{s}=|\gamma|\left(\alpha_{N}^{-1}+1\right) g_{s}=|\gamma| a^{-1} g_{s} .
$$

Also, we estimate

$$
\begin{aligned}
\frac{\left|\sum_{j=0}^{N} g_{a_{j}}\left(a_{j}-a_{j}^{\prime}\right)\right|}{g+s g_{s}} & \leq \frac{\left|a-\vec{a}^{\prime}\right| \sum_{j=0}^{N} s^{\alpha_{j}}}{\sum_{j=0}^{N} a_{j}\left(1+\alpha_{j}\right) s^{\alpha_{j}}} \leq \frac{\left|a-\vec{a}^{\prime}\right| \sum_{j=0}^{N} s^{\alpha_{j}}}{b_{0}(t)+b_{N}(t)\left(1+\alpha_{N}\right) s^{\alpha_{N}}} \\
& \leq\left|a-\vec{a}^{\prime}\right| \frac{C_{0} \chi(\vec{b}(t))(N+1)(1+s)^{\alpha_{N}}}{(1+s)^{\alpha_{N}}} \\
& \leq C_{0} \chi\left(\vec{a}, \vec{a}^{\prime}\right)\left|\vec{a}-\vec{a}^{\prime}\right| .
\end{aligned}
$$

Thus

$$
\begin{aligned}
& F^{\prime}(t) \geq(1-a) K(|\gamma(t)|, \vec{b}(t))\left|y-y^{\prime}\right|^{2} \\
& \quad-C_{0} \chi\left(\vec{a}, \vec{a}^{\prime}\right)\left|\vec{a}-\vec{a}^{\prime}\right| K(|\gamma(t)|, \vec{b}(t))|\gamma(t)|\left|y-y^{\prime}\right| .
\end{aligned}
$$

By the decrease of $K(\xi, \vec{a})$ in $\xi$ and $a_{j}$; and the increase of $K(\xi, \vec{a}) \xi$ in $\xi$, one has from (5.13) that

$$
\begin{aligned}
F^{\prime}(t) \geq(1-a) K\left(|y| \vee\left|y^{\prime}\right|, \vec{a} \vee \vec{a}^{\prime}\right)\left|y-y^{\prime}\right|^{2} \\
\\
-C_{0} \chi\left(\vec{a}, \vec{a}^{\prime}\right)\left|\vec{a}-\vec{a}^{\prime}\right| K\left(|y| \vee\left|y^{\prime}\right|, \vec{a} \wedge \vec{a}^{\prime}\right)\left(|y| \vee\left|y^{\prime}\right|\right)\left|y-y^{\prime}\right| .
\end{aligned}
$$

Combining with (5.11), we obtain (5.10).

When $N=0$ one has $\alpha_{N}=0, a=0, \vec{a}=a_{0}, \vec{a}^{\prime}=a_{0}^{\prime}, \vec{b}(t)=b(t)=b_{0}(t)$, $g(s, b)=b_{0}$, and $K(\xi, b)=b_{0}^{-1}$, and $g_{s}=0, g_{a_{0}}=1$. One has from (5.12)

$$
F^{\prime}(t) \geq K\left(|\gamma(t)|, b_{0}(t)\right)\left\{\left|y-y^{\prime}\right|^{2}-b_{0}(t)^{-1}\left|a_{0}-a_{0}^{\prime} \| \gamma(t)\right|\left|y-y^{\prime}\right|\right\} .
$$


Same as above, by the monotonicity of $K(\xi, \vec{a})$ and $K(\xi, \vec{a}) \xi$ one then obtains (5.10).

Let $g_{1}=g\left(s, \vec{a}^{(1)}\right)$ and $g_{2}=g\left(s, \vec{a}^{(2)}\right)$ be two functions of class $\operatorname{FP}(N, \alpha)$. Let $p_{k}$ $(k=1,2)$ be the solution of

$$
\frac{\partial p_{k}}{\partial t}=\nabla \cdot\left(K\left(\left|\nabla p_{k}\right|, \vec{a}^{(k)}\right) \nabla p_{k}\right)
$$

satisfying the Dirichlet boundary condition $(3.1 \mathrm{~d})$ on $\Gamma_{1}$ with the same data $\psi$, and the Neumann condition (3.1c) on $\Gamma_{2}$.

Let $p=p_{1}-p_{2}$, then

$$
\frac{\partial p}{\partial t}=\nabla \cdot\left(K\left(\left|\nabla p_{1}\right|, \vec{a}^{(1)}\right) \nabla p_{1}\right)-\nabla \cdot\left(K\left(\left|\nabla p_{2}\right|, \vec{a}^{(2)}\right) \nabla p_{2}\right) .
$$

Multiplying this equation by $p$ and integrating by parts over the domain yield

$$
\frac{1}{2} \frac{d}{d t} \int_{U} p^{2} d x=-\int_{U}\left(K\left(\left|\nabla p_{1}\right|, \vec{a}^{(1)}\right) \nabla p_{1}-K\left(\left|\nabla p_{2}\right|, \vec{a}^{(2)}\right) \nabla p_{1}\right) \cdot\left(\nabla p_{1}-\nabla p_{2}\right) d x .
$$

Let $H_{k}(\xi)=H\left(\xi, \vec{a}^{(k)}\right), K_{k}(\xi)=K\left(\xi, \vec{a}^{(k)}\right)$ for $k=1,2$.

The upper bound of the integral $\int_{U}\left|\nabla p_{k}(x, t)\right|^{2-a} d x$ established in (3.29) of Corollary 3.7 is needed in our later estimates, hence let

$$
\begin{aligned}
\bar{M}_{k}(t)= & 1+e^{-C_{1} t} \int_{U}\left|\nabla p_{k}(x, 0)\right|^{2-a} d x+\int_{U} \bar{p}_{k}^{2}(x, 0) d x \\
& +\int_{0}^{t} e^{-C_{1}(t-\tau)}\left(\Lambda_{1}(\tau)+G_{3}(\tau)\right) d \tau, \quad k=1,2
\end{aligned}
$$

and $\bar{M}=\bar{M}_{1}+\bar{M}_{2}$. One has

$$
\int_{U} K\left(\left|\nabla p_{k}\right|, \vec{a}^{(1)} \wedge \vec{a}^{(2)}\right)\left|\nabla p_{k}\right|^{2} d x \leq C+C \int_{U}\left|\nabla p_{k}\right|^{2-a} d x \leq C \bar{M}_{k},
$$

where $C$ depends on $\chi\left(\vec{a}^{(1)} \wedge \vec{a}^{(2)}\right)$ and $\chi\left(\vec{a}^{(k)}\right)$.

Proposition 5.3. For $t \geq 0$ one has

$$
\begin{aligned}
\int_{U}\left|p_{1}(x, t)-p_{2}(x, t)\right|^{2} d x \leq & \int_{U}\left|p_{1}(x, 0)-p_{2}(x, 0)\right|^{2} d x \\
& +C\left|\vec{a}^{(1)}-\vec{a}^{(2)}\right| \int_{0}^{t} \bar{M}(\tau) d \tau
\end{aligned}
$$

where $C>0$ depends on $N, \alpha_{N}$, and $\chi\left(\vec{a}^{(1)}, \vec{a}^{(2)}\right)$. Consequently, the solution $p(x, t ; \vec{a})$ depends continuously (in finite time intervals) on the initial data and the coefficient vector $\vec{a} \in R(N)$.

Proof. By (5.16) and the monotonicity of $K(|y|, \vec{a}) y$ established in Lemma 5.2 one immediately obtains

$$
\begin{aligned}
& \frac{1}{2} \frac{d}{d t} \int_{U}\left|p_{1}-p_{2}\right|^{2} d x \leq-(1-a) \int_{U} K\left(\left|\nabla p_{1}\right| \vee\left|\nabla p_{2}\right|, \vec{a}^{(1)} \vee \vec{a}^{(2)}\right)\left|\nabla p_{1}-\nabla p_{2}\right|^{2} d x \\
& +C\left|\vec{a}^{(1)}-\vec{a}^{(2)}\right| \int_{U} K\left(\left|\nabla p_{1}\right| \vee\left|\nabla p_{2}\right|, \vec{a}^{(1)} \wedge \vec{a}^{(2)}\right)\left(\left|\nabla p_{1}\right| \vee\left|\nabla p_{2}\right|\right)\left|\nabla p_{1}-\nabla p_{2}\right| d x,
\end{aligned}
$$


where the positive constant $C$ depends on $N, \alpha_{N}, \chi\left(\vec{a}^{(1)}, \vec{a}^{(2)}\right)$.

Applying Lemma 2.3 one estimates the first integral on the RHS of (5.20) and obtains

$$
\begin{aligned}
& \frac{1}{2} \frac{d}{d t} \int_{U}\left|p_{1}-p_{2}\right|^{2} d x \leq-C\left(\int_{U}\left|\nabla p_{1}-\nabla p_{2}\right|^{2-a} d x\right)^{\frac{2}{2-a}}\left(1+\left\|H_{1}\right\|_{L^{1}}+\left\|H_{2}\right\|_{L^{1}}\right)^{-a} \\
& +C\left|\vec{a}^{(1)}-\vec{a}^{(2)}\right| \int_{U} K\left(\left|\nabla p_{1}\right| \vee\left|\nabla p_{2}\right|, \vec{a}^{(1)} \wedge \vec{a}^{(2)}\right)\left(\left|\nabla p_{1}\right| \vee\left|\nabla p_{2}\right|\right)^{2} d x \\
& \leq-C\left(\int_{U}\left|\nabla p_{1}-\nabla p_{2}\right|^{2-a} d x\right)^{\frac{2}{2-a}}\left(1+\left\|H_{1}\right\|_{L^{1}}+\left\|H_{2}\right\|_{L^{1}}\right)^{-a} \\
& +C \chi\left(\vec{a}^{(1)} \wedge \vec{a}^{(2)}\right)\left|\vec{a}^{(1)}-\vec{a}^{(2)}\right| \int_{U}\left(\left|\nabla p_{1}\right| \vee\left|\nabla p_{2}\right|\right)^{2-a} d x \\
& \leq-C\left(\int_{U}\left|\nabla p_{1}-\nabla p_{2}\right|^{2-a} d x\right)^{\frac{2}{2-a}}\left(1+\left\|H_{1}\right\|_{L^{1}}+\left\|H_{2}\right\|_{L^{1}}\right)^{-a} \\
& +C \chi\left(\vec{a}^{(1)}, \vec{a}^{(2)}\right)\left|\vec{a}^{(1)}-\vec{a}^{(2)}\right| \int_{U}\left(\left|\nabla p_{1}\right|+\left|\nabla p_{2}\right|\right)^{2-a} d x .
\end{aligned}
$$

Therefore one obtains

$$
\begin{array}{r}
\frac{1}{2} \frac{d}{d t} \int_{U}\left|p_{1}-p_{2}\right|^{2} d x \leq-C\left(\int_{U}\left|\nabla p_{1}-\nabla p_{2}\right|^{2-a} d x\right)^{\frac{2}{2-a}}\left(1+\left\|H_{1}\right\|_{L^{1}}+\left\|H_{2}\right\|_{L^{1}}\right)^{-a} \\
+C\left|\vec{a}^{(1)}-\vec{a}^{(2)}\right| \int_{U}\left(\left|\nabla p_{1}\right|^{2-a}+\left|\nabla p_{2}\right|^{2-a}\right) d x
\end{array}
$$

where the positive constant $C$ depends on $N, \alpha_{N}, \chi\left(\vec{a}^{(1)}, \vec{a}^{(2)}\right)$.

Taking into account (5.18), one derives

$$
\begin{aligned}
& \frac{1}{2} \frac{d}{d t} \int_{U}\left|p_{1}-p_{2}\right|^{2} d x \leq-C\left(\int_{U}\left|\nabla p_{1}-\nabla p_{2}\right|^{2-a} d x\right)^{\frac{2}{2-a}}\left(\bar{M}_{1}(t)+\bar{M}_{2}(t)\right)^{-b} \\
& +C\left|\vec{a}^{(1)}-\vec{a}^{(2)}\right|\left(\bar{M}_{1}(t)+\bar{M}_{2}(t)\right) .
\end{aligned}
$$

Neglecting the negative term on the RHS and integrating this inequality in time yields (5.19).

Under the Degree Condition (2.28) and a growth constraint on the boundary data as $t \rightarrow \infty$, we obtain the Lyapunov stability, i.e., the continuous dependence (with respect to the $L^{2}$-norm) of the solution on the coefficient vector $\vec{a}$ uniformly in time $t$ over $[0, \infty)$.

We will use the upper bound of the integral $\int_{U}\left|\nabla p_{k}(x, t)\right|^{2-a} d x$ established in (3.30), hence define

$$
\begin{aligned}
M_{k}(t)= & 1+e^{-C_{1} t} \int_{U}\left|\nabla p_{k}(x, 0)\right|^{2-a} d x+\int_{U} \bar{p}_{k}^{2}(x, 0) d x \\
& +\int_{0}^{t} e^{-C_{1}(t-\tau)}\left(\Lambda_{2}(\tau)+G_{3}(\tau)\right) d \tau, \quad k=1,2,
\end{aligned}
$$

and $M(t)=M_{1}(t)+M_{2}(t)$. 
Proposition 5.4. Suppose $\operatorname{deg}(g) \leq 4 /(n-2)$. Then for $t \geq 0$,

$$
\begin{aligned}
& \int_{U}\left|p_{1}(x, t)-p_{2}(x, t)\right|^{2} d x \leq e^{-C_{1} \int_{0}^{t} M(\tau)^{-b}(\tau) d \tau} \int_{U}\left|p_{1}(x, 0)-p_{2}(x, 0)\right|^{2} d x \\
& +C\left|\vec{a}^{(1)}-\vec{a}^{(2)}\right| \int_{0}^{t} e^{-C_{1} \int_{\tau}^{t} M(\theta)^{-b}(\theta) d \theta} M(\tau) d \tau .
\end{aligned}
$$

Assume, in addition, that $\sup _{[0, \infty)} M(t)<\infty$ then

$$
\int_{U}\left|p_{1}(x, t)-p_{2}(x, t)\right|^{2} d x \leq e^{-C_{2} t} \int_{U}\left|p_{1}(x, 0)-p_{2}(x, 0)\right|^{2} d x+C_{3}\left|\vec{a}^{(1)}-\vec{a}^{(2)}\right|
$$

for all $t \geq 0$, and consequently

$$
\limsup _{t \rightarrow \infty} \int_{U}\left|p_{1}(x, t)-p_{2}(x, t)\right|^{2} d x \leq C_{3}\left|\vec{a}^{(1)}-\vec{a}^{(2)}\right| .
$$

Proof. Similar to the proof of Proposition 5.3 above, one has from (5.21)

$$
\frac{d}{d t} \int_{U}\left|p_{1}-p_{2}\right|^{2} d x \leq-C\left(\int_{U}\left|\nabla p_{1}-\nabla p_{2}\right|^{2-a} d x\right)^{\frac{2}{2-a}} M(t)+C\left|\vec{a}^{(1)}-\vec{a}^{(2)}\right| M(t) .
$$

Then by Poincare-Sobolev inequality:

$$
\frac{d}{d t} \int_{U}\left|p_{1}-p_{2}\right|^{2} d x \leq-C M(t) \int_{U}\left|p_{1}-p_{2}\right|^{2} d x+C\left|\vec{a}^{(1)}-\vec{a}^{(2)}\right| M(t) .
$$

Thus (5.22) follows by Gronwall's inequality.

The relations (5.23) and (5.24) are then obvious consequences. We omit further details.

Remark 5.5. In the above, $g_{1}$ and $g_{2}$ have the same length $(N+1)$ and exponent vector $\vec{\alpha}$. This is only for the sake of simplicity. The results apply also to the case when they have different lengths. Indeed, suppose $g_{k}$ has length $\left(N_{k}+1\right)$ with $\vec{\alpha}^{(k)}$ and $\vec{a}^{(k)}$, for $k=1,2$. Merge two vectors $\vec{\alpha}^{(1)}$ and $\vec{\alpha}^{(2)}$ to form a new common exponent vector $\vec{\alpha}$ with length $(N+1)$. Insert zero components into $\vec{a}^{(k)}$ to have new coefficient vector $\vec{A}^{(k)}$ with length $(N+1)$. Then one has $g\left(s, \vec{a}^{(k)} ; \vec{\alpha}^{(k)}\right)=$ $g\left(s, \vec{A}^{(k)} ; \vec{\alpha}\right)$ for all $s \geq 0$ and $k=1,2$.

For example, suppose $\vec{\alpha}^{(1)}=(0,1.5,3,4,5.8), \vec{a}^{(1)}=\left(a_{0}, a_{1}, a_{2}, a_{3}, a_{4}\right)$ and $\vec{\alpha}^{(2)}=(0,2,3,5.8), \vec{a}^{(2)}=\left(b_{0}, b_{1}, b_{2}, b_{3}\right)$. Then $\vec{\alpha}=(0,1.5,2,3,4,5.8), \vec{A}^{(1)}=$ $\left(a_{0}, a_{1}, 0, a_{2}, a_{3}, a_{4}\right)$ and $\vec{A}^{(2)}=\left(b_{0}, 0, b_{1}, b_{2}, 0, b_{3}\right)$.

We now discuss the uniform continuity of solution $p(x, t ; \vec{a})$ in $\vec{a}$.

Let $D$ be a compact set in $R(N)$, see (2.9). Define

$$
\hat{\chi}(D)=\max \{\chi(\vec{a}), \vec{a} \in D\} .
$$

Note that $\hat{\chi}(D) \in(0, \infty)$ and for any $\vec{a} \in D$, one has

$$
\hat{\chi}(D)^{-1} \leq \chi(\vec{a})^{-1} \leq \chi(\vec{a}) \leq \hat{\chi}(D) ;
$$

therefore the dependence of constants in Sect. 3 on individual $\chi(\vec{a})$ can now be replaced by the dependence on the common parameter $\hat{\chi}(D)$.

Let $\vec{a}^{(k)}$ belong to $D, k=1,2$. Set

$$
A_{*}=1+\int_{U}\left|\nabla p_{1}(x, 0)\right|^{2-a} d x+\int_{U} \bar{p}_{1}^{2}(x, 0) d x+\int_{U}\left|\nabla p_{2}(x, 0)\right|^{2-a} d x+\int_{U} \bar{p}_{2}^{2}(x, 0) d x,
$$




$$
\begin{aligned}
& \bar{\lambda}(t)=1+\int_{0}^{t} e^{-C_{1}(t-\tau)}\left(\Lambda_{1}(\tau)+G_{3}(\tau)\right) d \tau, \\
& \lambda(t)=1+\int_{0}^{t} e^{-C_{1}(t-\tau)}\left(\Lambda_{2}(\tau)+G_{3}(\tau)\right) d \tau,
\end{aligned}
$$

where $C_{1}$ depends on $N, \alpha_{N}$ and $\hat{\chi}(D)$.

Then

$$
\begin{aligned}
& \bar{M}(t) \leq A_{*} \bar{\lambda}(t), \\
& M(t) \leq A_{*} \lambda(t) .
\end{aligned}
$$

Proposition 5.3 and (5.26) lead to:

Theorem 5.6. Fix $N$ and $\vec{\alpha}$ with $\alpha_{N} \leq 4 /(n-2)$. Let $D$ be a compact subset of $R(N)$. Let $\vec{a}^{(k)}$ be in $D$ and $p_{k}(x, t)=p_{k}\left(x, t ; \vec{a}^{(k)}\right)$ be the corresponding solution to the IBVP (3.1a)-(3.1d) with Forchheimer polynomial $g\left(s, \vec{a}^{(k)}\right)$, for $k=1,2$. For $t \geq 0$ one has

$$
\begin{aligned}
\int_{U}\left|p_{1}(x, t)-p_{2}(x, t)\right|^{2} d x \leq & \int_{U}\left|p_{1}(x, 0)-p_{2}(x, 0)\right|^{2} d x \\
& +C A_{*}\left|\vec{a}^{(1)}-\vec{a}^{(2)}\right| \int_{0}^{t} \bar{\lambda}(\tau) d \tau
\end{aligned}
$$

where $C>0$ depends on $N, \alpha_{N}$, and $\hat{\chi}(D)$.

Proposition 5.4 leads to:

Theorem 5.7. Fix $N$ and $\vec{\alpha}$ with $\alpha_{N} \leq 4 /(n-2)$. Let $D$ be a compact subset of $R(N)$. Let $\vec{a}^{(k)}$ be in $D$ and $p_{k}(x, t)=p_{k}\left(x, t ; \vec{a}^{(k)}\right)$ be the corresponding solution to the IBVP (3.1a)-(3.1d) with Forchheimer polynomial $g\left(s, \vec{a}^{(k)}\right)$, for $k=1,2$.

(i) One has for $t \geq 0$ that

$$
\begin{aligned}
& \int_{U}\left|p_{1}(x, t)-p_{2}(x, t)\right|^{2} d x \leq e^{-C_{1} B_{*} \int_{0}^{t} \lambda(\tau)^{-b} d \tau} \int_{U}\left|p_{1}(x, 0)-p_{2}(x, 0)\right|^{2} d x \\
& +C_{2} A_{*}\left|\vec{a}^{(1)}-\vec{a}^{(2)}\right| \int_{0}^{t} e^{-C_{1} B_{*} \int_{\tau}^{t} \lambda(\theta)^{-b} d \theta} \lambda(\tau) d \tau
\end{aligned}
$$

where $B_{*}=A_{*}^{-b}$, and the constants $C_{1}, C_{2}$ depend on $N, \alpha_{N}, \hat{\chi}(D)$.

(ii) Assume, in addition, that $\lambda(t) \leq C_{3}$ for all $t \geq 0$ then

$$
\begin{aligned}
\int_{U}\left|p_{1}(x, t)-p_{2}(x, t)\right|^{2} d x \leq & e^{-C_{4} B_{*} t} \int_{U}\left|p_{1}(x, 0)-p_{2}(x, 0)\right|^{2} d x \\
& +C_{5} A_{*}^{1+b}\left|\vec{a}^{(1)}-\vec{a}^{(2)}\right|,
\end{aligned}
$$

where $C_{4}, C_{5}$ depend on $N, \alpha_{N}, \hat{\chi}(D)$ and $C_{3}$. Consequently

$$
\limsup _{t \rightarrow \infty} \int_{U}\left|p_{1}(x, t)-p_{2}(x, t)\right|^{2} d x \leq C_{5} A_{*}^{1+b}\left|\vec{a}^{(1)}-\vec{a}^{(2)}\right| .
$$

(iii) Particularly, if $\lim _{t \rightarrow \infty} \lambda(t)=L \in(0, \infty)$ then

$$
\limsup _{t \rightarrow \infty} \int_{U}\left|p_{1}(x, t)-p_{2}(x, t)\right|^{2} d x \leq C_{6} L^{1+b} A_{*}^{1+b}\left|\vec{a}^{(1)}-\vec{a}^{(2)}\right|,
$$

where $C_{6}$ depends on $N, \alpha_{N}$ and $\hat{\chi}(D)$. 
Proof. Parts (i) and (ii) are direct consequences of Proposition 5.4 and (5.27).

For (iii), first note that $\lim _{t \rightarrow \infty} \int_{0}^{t} \lambda(\tau)^{-b} d \tau=\infty$; then apply the L'Hôpital Rule to the term $A_{*} e^{-C B_{*} \int_{0}^{t} \lambda(\theta)^{-b} d \theta} \int_{0}^{t} e^{C B_{*} \int_{0}^{\tau} \lambda(\theta)^{-b} d \theta} \lambda(\tau) d \tau$ when taking the limit of the RHS of (5.29) as $t \rightarrow \infty$.

\section{Appendix A}

We will prove an estimate for solutions of a particular differential inequality which is used in Sect. 3 .

Definition A.1. Given $f(t)$ defined on an interval I. A function $F(t)$ is called an (upper) envelop of $f(t)$ on $I$ if $F(t) \geq f(t)$ for all $t \in I$. We denote an envelop function of $f(t)$ by $\operatorname{Env}(f)$.

Lemma A.2. Suppose

$$
y^{\prime} \leq-A y^{\alpha}+f(t)
$$

for all $t>0$, with $A, \alpha>0$ and $y(t), f(t) \geq 0$.

Let $F(t)$ be a continuous, increasing envelop of $f(t)$ on $[0, \infty)$. Then one has

$$
y(t) \leq y(0)+A^{-1 / \alpha} F(t)^{1 / \alpha}, \quad \forall t \geq 0 .
$$

Proof. One has form (A.1) that

$$
y^{\prime} \leq-A y^{\alpha}+F(t), \quad \forall t>0 .
$$

Note that $-A y^{\alpha}+F(t) \leq 0$ iff $y(t) \geq B F(t)^{1 / \alpha}$, where $B=A^{-1 / \alpha}$.

Claim: For any $\delta>0$,

$$
y(t) \leq y(0)+\delta+B F(t)^{1 / \alpha}, \quad \forall t \geq 0 .
$$

Then letting $\delta \rightarrow 0$ in (A.4) yields (A.2).

Proof of (A.4): Suppose the statement is false. Then by using the function $g(t)=y(t)-y(0)-B F(t)^{1 / \alpha}$ one can show that there are $t_{1}<t_{2}$ such that

$$
\begin{gathered}
y\left(t_{1}\right)=y(0)+B F\left(t_{1}\right)^{1 / \alpha}, \\
y\left(t_{2}\right)=y(0)+\delta+B F\left(t_{2}\right)^{1 / \alpha},
\end{gathered}
$$

and

$$
y(t) \geq y(0)+B F(t)^{1 / \alpha}, \quad \forall t \in\left[t_{1}, t_{2}\right) .
$$

The last inequality yields $y^{\prime}(t) \leq 0$ for all $t \in\left[t_{1}, t_{2}\right)$, hence

$$
y\left(t_{2}\right) \leq y\left(t_{1}\right) \leq y(0)+B F\left(t_{1}\right)^{1 / \alpha} \leq y(0)+B F\left(t_{2}\right)^{1 / \alpha}<y\left(t_{2}\right),
$$

which is a contradiction. Therefore one has (A.4).

In the following, we presents a construction of smooth, increasing envelop functions.

Let $f(t) \geq 0$ be defined on $[0, \infty)$ and locally bounded. Define for $t \geq 0$

$$
F_{1}(t)=\sup \{f(s), 0 \leq s \leq t\} .
$$

and for $t<0$

$$
F_{1}(t)=F_{1}(0)=f(0) .
$$

Then $F_{1}(t)$ is an increasing function on $\mathbb{R}$ and $F_{1}(t) \geq f(t)$ for all $t \geq 0$. 
For $0<\varepsilon<1$, define the mollifier

$$
F^{\varepsilon}(t)=\int_{-\infty}^{\infty} \eta_{\varepsilon}(t-\tau) F_{1}(\tau+\varepsilon) d \tau=\int_{-\infty}^{\infty} \eta_{\varepsilon}(\tau) F_{1}(t-\tau+\varepsilon) d \tau
$$

Then $F^{\varepsilon}(t)$ is an increasing, smooth function and for $t \geq 0$ one has

$$
F^{\varepsilon}(t)=\int_{t-\varepsilon}^{t+\varepsilon} \eta_{\varepsilon}(t-\tau) F_{1}(\tau+\varepsilon) d \tau \geq \int_{t-\varepsilon}^{t+\varepsilon} \eta_{\varepsilon}(t-\tau) F_{1}(t) d \tau=F_{1}(t) \geq f(t)
$$

If $f(t)$ is a $L_{l o c}^{\infty}([0, \infty))$ function, then $F^{\varepsilon}(t) \geq f(t)$ a.e. on $[0, \infty)$; and consequently, this holds at the points $t$ which $f$ is continuous.

Acknowledgment. The authors would like to thank Eugenio Aulisa for his stimulating discussions. This research is supported by NSF Grant No. DMS-0908177.

\section{REFERENCES}

[1] E. Aulisa, L. Bloshanskaya, L. Hoang, A. I. Ibragimov, Analysis of Generalized Forchheimer Flows of Compressible Fluids in Porous Media, J. Math. Phys. 50, 103102 (2009), DOI:10.1063/1.3204977.

[2] E. Aulisa, A. I. Ibragimov, J. R. Walton, A new method for evaluation the productivity index for non-linear flow, SPE Journal, SPE Journal, Volume 14, Number 4, 693-706, (2009), DOI:10.2118/108984-PA.

[3] E. Aulisa, A. I. Ibragimov, P. P. Valkó, J. R. Walton, Mathematical Frame-Work For Productivity Index of The Well for Fast Forchheimer (non-Darcy) Flow in Porous Media, Mathematical Models and Methods in Applied Sciences (M3AS), 8, 1241-1275, (2009), DOI:10.1142/S0218202509003772.

[4] M. T. Balhoff, A. Mikelic, M. F. Wheeler, Polynomial Filtration Laws for Low Reynolds Number Flows Through Porous Media, Transport in Porous Media, in press (2009), DOI:10.1007/s11242-009-9388-z.

[5] J. Bear, Dynamics of Fluids in Porous Media, Dover Publications, Inc., New York, (1972).

[6] J. Chadam, Y. Qin, Spatial decay estimates for flow in a porous medium, SIAM J. Math. Anal., Vol. 28, No. 4, 808-830 (1997).

[7] J. Jr. Douglas, P. J. Paes-Leme, T. Giorgi, Generalized Forchheimer flow in porous media, Boundary value problems for partial differential equations and applications, 99-111, RMA Res. Notes Appl. Math., 29, Masson, Paris, (1993).

[8] L. J. Durlofsky, M. Karimi-Fard, Detailed near-well Darcy-Forchheimer flow modeling and upscaling on unstructured 3D grids, SPE 118999, (2009), DOI:10.2118/118999-MS.

[9] E. Ewing, R. Lazarov, S. Lyons, D. Papavassiliou, Numerical well model for non Darcy flow, Comp. Geosciences, 3, 3-4, 185-204 (1999).

[10] P. Forchheimer, Wasserbewegung durch Boden Zeit, Ver. Deut. Ing. 45, (1901).

[11] D. Li, T. W. Engler, Literature Review on Correlations of the Non-Darcy Coefficient, SPE, published online (2001), DOI:10.2118/70015-MS.

[12] V. G. Mazya, Sobolev spaces, Translated from the Russian by T. O. Shaposhnikova. Springer Series in Soviet Mathematics. Springer-Verlag, Berlin, (1985).

[13] E. Marusic-Paloka, A. Mikelic The derivation of a nonlinear filtration law including the inertia effects via homogenization Nonlinear Analysis 42, 97-137, (2000).

[14] C. Miranda, Partial Differential Equations of Elliptic Type, Translated by Zane C. Motteler, Springer-Verlag New-York, Heidelberg, Berlin, (1970).

[15] M. Muskat, The flow of homogeneous fluids through porous media, McGraw-Hill Book Company, Inc., New York and London, (1937).

[16] E.-J. Park, Mixed finite element methods for generalized Forchheimer flow in porous media, Numer. Methods Partial Differential Equations 21, no. 2, 213-228 (2005).

[17] L. E. Payne, B. Straughan, Convergence and Continuous Dependence for the BrinkmanForchheimer Equations, Studies in Applied Mathematics, 102, 419-439 (1999). 
[18] L. E. Payne, J. C. Song, B. Straughan, Continuous dependence and convergence results for Brinkman and Forchheimer models with variable viscosity, R. Soc. Lond. Proc. Ser. A Math. Phys. Eng. Sci. 455, no. 1986, 2173-2190 (1999).

[19] L. E. Payne, J. C. Song, Spatial decay estimates for the Brinkman and Darcy flows in a semi-infinite cylinder, Contin. Mech. Thermodyn. 9, no. 3, 175-190 (1997).

[20] L. E. Payne, B. Straughan, Stability in the initial-time geometry problem for the Brinkman and Darcy equations of flow in porous media, J. Math. Pures Appl. (9) 75, no. 3, 225-271 (1996).

[21] P. Polubarinova-Kochina, Theory of Ground Water Movement, translated from the Russian by J. M. Roger De Wiest, Princeton University Press, (1962).

[22] D. Ruth, H. Ma, On the Derivation of the Forchheimer Equation by Means of the Averaging Theorem, Transport in Porous Media, 7: 255-264 (1992).

[23] K. R. Rajagopal, On a hierarchy of approximate models for flows of incompressible fluids through porous solids, Mathematical Models and Methods in Applied Sciences, Vol. 17, No. 2, 215-252 (2007).

[24] B. Straughan, Stability and wave motion in porous media, Applied Mathematical Sciences, 165, Springer, New York, (2008).

[25] S. Whitaker, The Forchheimer Equation: A Theoretical Development, Transport in Porous Media 25: 27-61 (1996).

Department of Mathematics and Statistics, Texas Tech University, Box 41042, LubBOCK, TX 79409-1042, U. S. A.

E-mail address: luan.hoang@ttu.edu

E-mail address: akif.ibraguimov@ttu.edu ( ${ }^{\dagger}$ Corresponding author) 